\title{
Spectral diversity and photometric behavior of main-belt and near-Earth vestoids and (4) Vesta: A study in preparation for the Dawn encounter
}

\author{
Michael D. Hicks ${ }^{a}{ }^{*}$, Bonnie J. Buratti ${ }^{a}$, Kenneth J. Lawrence ${ }^{a}$, John Hillier ${ }^{b}$, Jian-Yang Li ${ }^{c}$, Vishnu Reddy ${ }^{d}$, \\ Stefan Schröder ${ }^{\mathrm{d}}$, Andreas Nathues ${ }^{\mathrm{d}}$, Martin Hoffmann ${ }^{\mathrm{d}}$, Lucille Le Corre ${ }^{\mathrm{d}}$, Rene Duffard ${ }^{\mathrm{e}}$, Hai-Bin Zhao ${ }^{\mathrm{f}}$, \\ Carol Raymond $^{\text {a }}$, Christopher Russell ${ }^{g}$, Thomas Roatsch ${ }^{\mathrm{h}}$, Ralf Jaumann ${ }^{\mathrm{h}}$, Heath Rhoades ${ }^{\mathrm{a}}$, \\ Deronda Mayes $^{a}$, Tzitlaly Barajas ${ }^{i}$, Thien-Tin Truong ${ }^{i}$, James Foster ${ }^{i}$, Amanda McAuley ${ }^{i}$
}

\footnotetext{
a Jet Propulsion Laboratory, California Inst. of Technology, 4800 Oak Grove Drive 183-501, Pasadena, CA 91109, USA

${ }^{\mathrm{b}}$ Grays Harbor College, 1620 Edward P Smith Drive, Aberdeen, WA 98520-7599, USA

${ }^{\mathrm{c}}$ Department of Astronomy, University of Maryland, College Park, MD 20742, USA

${ }^{\mathrm{d}}$ Max Planck Institut für Sonnensystemforschung, 37191 Katlenburg-Lindau, Germany

e Instituto de Astrofisica de Andalucia, CSIC, C/Bajo de Huetor, 50, Granada 18008, Spain

${ }^{\mathrm{f}}$ Purple Mountain Observatory, Chinese Academy of Sciences, Nanjing 210008, China

$\mathrm{g}$ Institute of Geophysics and Planetary Physics, University of California Los Angeles, Los Angeles, CA 90024-1567, USA

${ }^{\mathrm{h}}$ Institute of Planetary Research, Deutsches Zentrum für Luft- und Raumfahrt, Berlin 80302, Germany

${ }^{i}$ California State University Los Angeles, State University Drive, Los Angeles, CA 90032, USA
}

\section{A R T I C L E I N F O}

\section{Article history:}

Received 20 September 2012

Revised 8 November 2013

Accepted 8 November 2013

Available online 24 February 2014

\section{Keywords:}

Asteroid Vesta

Near-Earth objects

Photometry

Spectroscopy

\begin{abstract}
A B S T R A C T
In anticipation of the Dawn Mission to 4 Vesta, we conducted a ground-based campaign of Bessel BVRI filter photometry of five V-type near-Earth asteroids over a wide range of solar phase angles. We also obtained medium-resolution optical spectroscopy $(0.38 \mu \mathrm{m}<\lambda<0.92 \mu \mathrm{m} ; R \sim 500)$ of sixteen near-Earth and mainbelt $\mathrm{V}$-type asteroids in order to investigate their spectral diversity and to draw connections between spacecraft data of Vesta and V-type asteroids. Our disk-integrated photometry extended the excursion in solar phase angle beyond the maximum of $24^{\circ}$ available from Earth for Vesta to $87^{\circ}$, which is more typical of the geometry during the Dawn approach and mapping phases. The majority of our broad-band observations were obtained at the JPL 0.6-m Table Mountain Observatory but multiple nights were also contributed by the Calar Alto $1.2-\mathrm{m}$ and $2.2-\mathrm{m}$ telescopes, as well as by the Purple Mountain 1-m Schmidt. Our results include a determination of rotation periods for 4 asteroids, identification of a binary candidate and four new V-type asteroids, including a confirmation of two main-belt V-type asteroids beyond the Jupiter 1:3 resonance (Cruikshank, D.P., Tholen, D.J., Bell, J.F., Hartmann, W.K., Brown, R.H. [1991]. Icarus 89, 1-13; Lazzaro, D. et al. [2000]. Science 288, 2033-2035; Roig, F., Gil-Hutton, R. [2006]. Icarus 183(2), 411-419; Moskovitz, N.A., Jedicke, R., Gaidos, E., Willman, M., Nesvorný, D., Fevig, R., Ivezić, Ž. [2008]. Icarus 198, 77-90). This latter finding supports the hypothesis that some vestoids may be crustal fragments of a disrupted basaltic parent body compositionally similar to 4 Vesta. We also obtained rotationally resolved medium resolution spectra of Vesta during the Dawn orbit insertion phase, which will be valuable for calibration and comparison of spacecraft data. Modeling of a composite V-type asteroid phase curve yielded a generic photometric model for $\mathrm{V}$ asteroids. We also find that a significant amount of the spectral diversity in the $\mathrm{V}$ class comes from changes in solar phase angle. A fit of a composite solar phase curve containing our vestoid observations, previously published groundbased observations of Vesta, and early disk-integrated Dawn observations show important differences with other asteroids. The macroscopic surface roughness of V-type asteroids is significantly larger than that of C-type or S-types (Helfenstein, P., Veverka, J. [1989]. Physical characterization of asteroid surfaces from photometric analysis. In: Binzel, R., Gehrels, T., Matthews, M.S. (Eds.), Asteroids II. University of Arizona Press, Tucson, pp. 557-593). This result is consistent with radar studies showing that igneous rocky asteroids - the $\mathrm{E}$ and V types - exhibit the largest surface roughness (Benner, L. et al. [2008]. Icarus 198, 294-304). The effects of what appears to be space weathering can be largely explained by phase reddening in our collection of
\end{abstract}

\footnotetext{
* Corresponding author. Address: Jet Propulsion Laboratory, MS 183-501, 4800 Oak Grove Drive, Pasadena, CA 91109, USA.

E-mail addresses: Michael.Hicks@jpl.nasa.gov (M.D. Hicks), jyli@astro.umd.edu (J.-Y. Li), duffard@iaa.es (R. Duffard).
} 
V-type NEOs, but our finding that smaller vestoids, which have shorter lifetimes, are more similar to Vesta suggests that some type of alteration of the surface through time occurs. Our observations confirm that the south polar region of Vesta has a more diogenitic composition than its equatorial regions. The south pole, which is dominated by a large impact feature, thus may offer a view into the interior of Vesta. We derive a visible phase integral of $0.44 \pm 0.02$ and a corresponding Bond albedo of $0.15 \pm 0.03$ from our composite V-type asteroid solar phase curve.

(c) 2014 Published by Elsevier Inc.

\section{Introduction}

The Dawn Mission is part of NASA's Discovery Program. Launched September 27, 2007, the spacecraft began the detailed study of the Main Belt Asteroid 4 Vesta with orbit insertion on July 17, 2011 and continuation of its orbital mission for 1 year. The main goals of Dawn's in-depth study are to understand the evolution of this protoplanet and the role of water in its history; to derive Vesta's bulk properties; to model its surface composition and geologic landforms, and to understand its relationship with the terrestrial HED meteorites and the near-Earth vestoids (Kelley et al., 2003; Binzel et al., 2004; Russell et al., 2012), both of which are believed to originate from Vesta. In preparation for the Dawn encounter, a ground-based observing campaign to study the spectrophotometric properties of vestoids was undertaken. One important component of this program was the gathering of photometric observations of vestoids over a complete range in solar phase angles. Because Vesta is in the Main Belt, the maximum excursion in solar phase angle is limited to $\sim 25^{\circ}$. The majority of the observations to be obtained by Dawn are at larger solar phase angles. No project data products or scientific results - mosaics for both public consumption and for geophysical studies, composition based on spectroscopic band identifications, topographic analysis based on photoclinometry, shape models, etc. - can be of very high fidelity without careful photometric modeling and correction.

One primary goal of this study was to derive a surface phase function for Vesta and vestoids to be in place at the start of the nominal mapping mission. A related goal is to quantify the effects of solar phase reddening in V-type asteroids, and to understand how much spectral diversity is due to the effects of changing radiance and viewing geometry. The final goal of our study is to explore the variability in the optical reflectance of vestoids to better understand their relationship to Vesta and the origin and dynamical history of the Near-Earth Objects (NEOs) and the terrestrial meteorites in general. Our measurements also resulted in a number of subsidiary findings, including four new rotation curves for near-Earth vestoids, identification of several new $\mathrm{V}$-types and a binary candidate, and constraints on a pole position for one vestoid. We also obtained hundreds of medium resolution spectra of Vesta over a full rotation period at the same time as the Dawn orbit insertion, which will enable better instrument calibration and provide context for ground-based observations. Finally, we did extensive photometric modeling of our observations to yield a general V-type model that can be compared to similar models developed for S-type and C-type asteroids (Helfenstein and Veverka, 1989).

\section{Spectral observations: acquisition and reduction}

Medium resolution optical spectrophotometry $\left(R \sim 300 ; \lambda_{\text {MIN }}-\right.$ $\left.\sim 0.38 \mu \mathrm{m} ; \lambda_{\text {MAX }} \sim 0.95 \mu \mathrm{m}\right)$ of 7 near-Earth and 10 main-belt V-type asteroids were obtained at the Palomar Mountain 200-in. telescope (P200) equipped with a facility dual-channel long-slit CCD spectrometer (the "Double-Spec" or DBSP; Oke and Gunn,
1982). The observing circumstances are listed in Table 1 with orbital elements, absolute magnitude, etc., as provided by the JPL Horizons database. The Near-Earth Objects (NEOs) in our sample were initially targeted in our ongoing survey of Potentially Hazardous Asteroids (PHAs), low- $\Delta V$ NEOs, and asteroids scheduled for radar observation. Their V-type taxonomy was discovered serendipitously as we began to collect data on them. For our main-belt spectral targets, we used the broad-band colors archived in the 4th release of the Sloan Digital Sky Survey Moving Object Catalog (Ivezic et al., 2002) in comparison to the V-type asteroids contained in the SMASS II survey to identify potential V-type asteroids available during our scheduled observing runs, which we confirmed with our Palomar spectroscopy. In this way we have been able to build a self-consistent, unbiased database of V-type asteroids over a wide range of sizes and orbital distribution, as illustrated in Fig. 1. A number of our asteroids targeted as V-type by their optical SDSS colors have been studied by other observers, including 4055 Magellen (Cruikshank et al., 1991), 5599 (1991 SG1) (Roig and Gil-Hutton, 2006). Although 7472 Kumakiri exhibits optical colors compatible with V-type classification, the inclusion of near-IR data revealed a reflectance spectrum more similar to the O-type classification of 3628 Božněmcová (Burbine et al., 2011). Additionally, Roig and Gil-Hutton (2006), Moskovitz et al. (2008), and Marchi et al. (2010) have used SDSS colors to identify basaltic asteroids in the main-belt.

With the Palomar DBSP the night sky and object are first imaged on the slit before being divided by a dichroic filter into blue and red beams which are then dispersed and reimaged with individual grating and camera set-ups. In addition to our target asteroids, spectra of solar analog stars over a wide range of airmass were obtained throughout each night. Wavelength calibrations were accomplished with arc-lamp exposures and flat-fields were taken using the illuminated dome. Our individual spectral exposures on the NEOs were $300 \mathrm{~s}$. Between exposures we recentered on the object and updated the object rates of motion if needed. Typical pointing drifts were on the order of 1 arcsec. Throughout our campaign we used a 6 arcsec wide slit and kept the tailpiece of the telescope rotated to match the parallactic angle. The effective exposure times for our fainter targets were approximately $1 \mathrm{~h}$.

The spectral data reduction proceeded in a standard manner (Hicks and Buratti, 2004), using IRAF and custom built code. The two channels of the spectrograph were separately analyzed and recombined into a composite spectrum. The platescales of the red and blue cameras are 0.468 and 0.624 arcsec pixel $^{-1}$, respectively. After flat-fielding, for each exposure a 21-pixel wide object window was defined in the spatial dimension about the object centerline with 5 pixel sky windows immediately adjacent. For each exposure, after flat-fielding, a 21-pixel wide object window was defined in the spatial dimension about the object's centerline with a 5 pixel sky window immediately adjacent for each exposure. A linear fit to the sky windows was subtracted from each row to remove scattered light and night-sky emissions. The digital counts were summed across the object into a one-dimensional spectrum. Solar analog stars were reduced in a similar way and coadded to match the airmass of our asteroids and ratioed to produce relative 
Table 1

Orbital elements and observing circumstances: Palomar spectroscopy targets.

\begin{tabular}{|c|c|c|c|c|c|c|c|c|c|}
\hline Object & UT date & $a(\mathrm{AU})$ & $e$ & $i\left(^{\circ}\right)$ & $q(\mathrm{AU})$ & $H$ (mag) & $\alpha\left(^{\circ}\right)$ & $V($ mag $)$ & Obs. $^{a}$ \\
\hline 2004 LV3 & 20081227.19 & 1.23 & 0.28 & 35.3 & 0.89 & 18.8 & 84.3 & 15.9 & $\mathrm{MH}, \mathrm{KL}$ \\
\hline 2003 EF54 & 20090825.23 & 1.61 & 0.47 & 3.0 & 0.85 & 20.0 & 43.1 & 16.2 & $\mathrm{MH}, \mathrm{KL}, \mathrm{TB}, \mathrm{AM}$ \\
\hline 8566 (1996 EN) & 20090825.49 & 1.51 & 0.43 & 38.0 & 0.86 & 16.5 & 93.5 & 16.3 & $\mathrm{MH}, \mathrm{KL}, \mathrm{TB}, \mathrm{AM}$ \\
\hline 2004 FG11 & 20100407.49 & 1.59 & 0.44 & 3.1 & 0.89 & 20.9 & 62.7 & 17.7 & $\mathrm{MH}, \mathrm{KL}$ \\
\hline 4055 Magellan & 20100810.16 & 1.82 & 0.33 & 23.2 & 1.23 & 14.8 & 52.6 & 16.0 & $\mathrm{BB}, \mathrm{MH}$ \\
\hline $2010 \mathrm{MF} 1$ & 20100810.18 & 2.50 & 0.59 & 9.1 & 1.02 & 19.7 & 81.6 & 18.2 & $\mathrm{BB}, \mathrm{MH}$ \\
\hline 199432 (1998 FL71) & 20100810.41 & 1.38 & 0.08 & 5.8 & 2.18 & 14.0 & 4.6 & 17.4 & $\mathrm{BB}, \mathrm{MH}$ \\
\hline 35965 (1999 LH13) & 20100810.44 & 2.32 & 0.14 & 6.8 & 2.01 & 15.0 & 15.1 & 17.8 & $\mathrm{BB}, \mathrm{MH}$ \\
\hline 7472 Kumakiri & 20100810.47 & 3.02 & 0.10 & 9.9 & 2.71 & 11.9 & 12.5 & 17.3 & $\mathrm{BB}, \mathrm{MH}$ \\
\hline $19165(1991 \mathrm{CD})$ & 20100810.49 & 2.29 & 0.07 & 7.8 & 2.12 & 13.7 & 20.9 & 17.6 & $\mathrm{BB}, \mathrm{MH}$ \\
\hline 12073 Larimer & 20100901.24 & 2.42 & 0.08 & 6.2 & 2.22 & 14.1 & 14.0 & 17.3 & $\mathrm{BB}, \mathrm{MH}$ \\
\hline 2247 Hiroshima & 20100901.47 & 2.45 & 0.11 & 5.9 & 2.18 & 13.9 & 22.9 & 17.6 & $\mathrm{BB}, \mathrm{MH}$ \\
\hline 6093 Makoto & 20100901.51 & 2.48 & 0.14 & 6.2 & 2.14 & 13.2 & 27.0 & 17.3 & $\mathrm{BB}, \mathrm{MH}$ \\
\hline 1991 SG1 & 20101009.43 & 3.17 & 0.08 & 9.9 & 2.92 & 12.8 & 19.4 & 16.0 & $\mathrm{MH}, \mathrm{KL}$ \\
\hline 1999 VO6 & 20101009.49 & 1.14 & 0.30 & 40.1 & 0.80 & 16.9 & 82.7 & 15.3 & $\mathrm{MH}, \mathrm{KL}$ \\
\hline 2005 GC120 & 20101212.25 & 1.19 & 0.60 & 16.5 & 0.48 & 19.6 & 40.6 & 16.9 & $\mathrm{MH}, \mathrm{KL}$ \\
\hline
\end{tabular}

a Observer key: $\mathrm{AM}=$ Amanda McAuley, $\mathrm{BB}=$ Bonnie Buratti, $\mathrm{KL}=$ Kenneth Lawrence, $\mathrm{MH}=$ Michael Hicks, TB = Tzitlaly Barajas.

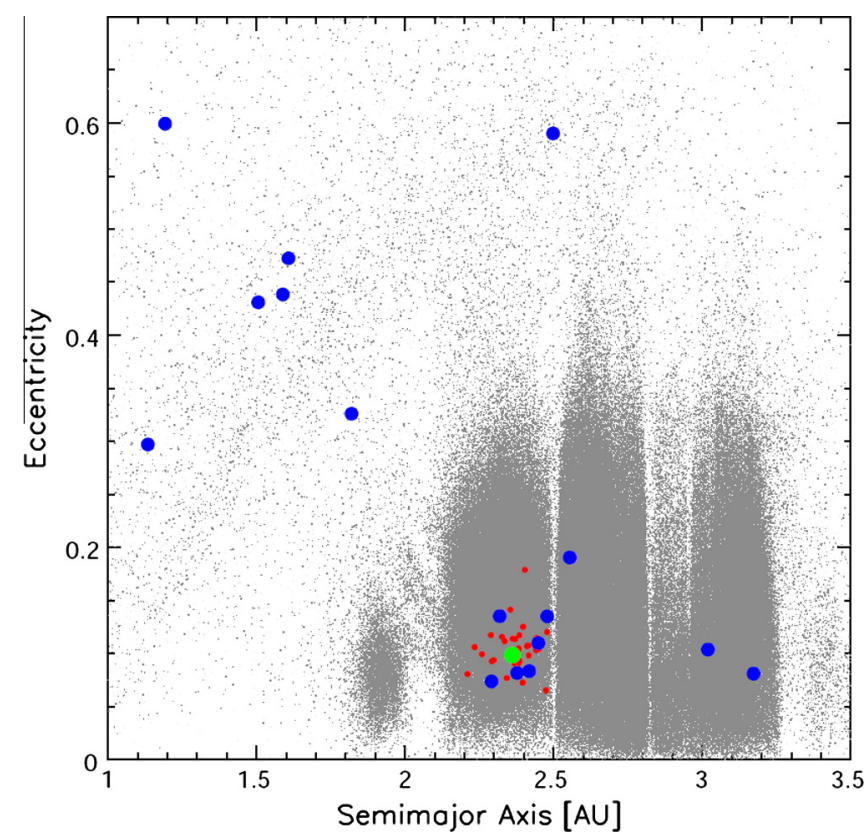

Fig. 1. Osculating elements for the V-type asteroids observed at Palomar (blue), archived V-type asteroids in the SMASSII spectral database (red), and 4 Vesta (green). For reference, the proper elements of the known Main Belt asteroids are shown (Nesvorny, as archived on http://hamilton.dm.unipi.it/astdys). (For interpretation of the references to color in this figure legend, the reader is referred to the web version of this article.)

reflectance. Our exposures were obtained in rapid succession and individual frames in each sequence were ratioed by their sum. Changes in slope or flux in the ratios would suggest problems with differential refraction or changing extinction but in no cases was it required to omit any single spectral frame from the sums. Similarly, we were able to cross reference our solar comparison stars taken on various nights with the well accepted solar analog 16 Cyg B. Fig. 2 illustrates the results of our vestoid spectroscopy. The relative reflectance for these 16 objects have been normalized to unity at $0.55 \mu \mathrm{m}$, offset for clarity, and presented in order of increasing $1 \mu \mathrm{m}$ band depth.

\section{Spectral observations: results}

At optical wavelengths, the spectra of Vesta and the vestoids can be characterized by the depth of the $1 \mu \mathrm{m}$ absorption feature and the continuum slope near $0.6 \mu \mathrm{m}$. These quantities can be used to explore the spectral diversity of V-type asteroids and compared directly with Vesta. The P200 spectra do not cover fully the extent of the $1 \mu \mathrm{m}$ band; therefore, we used the reflectance at $0.85 \mu \mathrm{m}$ to compute a pseudo-band depth. After smoothing our spectra with a $0.01 \mu \mathrm{m}$ wide boxcar median filter we found the maximum reflectance $R_{M A X}$ and defined the pseudo-band depth $D=\left(R_{\text {MAX }}-R_{0.85 \mu \mathrm{m}}\right) / R_{\text {MAX }}$. The spectral slope $S$ was defined as the percent change across $0.6-0.7 \mu \mathrm{m}$. Table 2 lists the spectral slope and pseudo-band depth for our P200 targets as well as for the V-type asteroids identified in the SMASSII asteroid spectral survey database (Bus and Binzel, 2002a); the results are plotted in Fig. 3. The distribution of spectral slopes and pseudo-band depths for the P200 and SMASSII populations agree. The P200 and SMASSII populations on average show steeper slopes and greater pseudoband depth than 4 Vesta. The lower four panels of Fig. 3 explore possible correlations of $S$ and $D$ with absolute magnitude $H$ (a proxy for size) and perihelion distance $q$ (position within the asteroid belt). For our P200 vestoids, no correlations of psuedo-band depth $D$ were found with respect to absolute magnitude, perihelion distance, or solar phase angle $(\alpha)$, as summarized in Table 3. However, significant correlations of spectral slope $S$ were found with respect to absolute magnitude $(\sigma=4.8)$, perihelion distance ( $\sigma=2.7)$, and solar phase angle $(\sigma=1.8)$. As illustrated in the lower left-hand panel of Fig. 2, the small vestoids tend to have spectral slopes more compatible with that of 4 Vesta. This result may suggest evidence for some type of space weathering: small NEOs have shorter collisional lifetimes and may reflect younger, unaltered surfaces. Close approaches of NEOs to the Earth-Moon system may lead to a gravitational shaking that refreshes the near surface regolith (Nesvorný et al., 2005; Marchi et al., 2006; Binzel et al., 2010).

The taxonomy of the near-Earth asteroids presented in this paper was determined during our ongoing spectral survey of planetary radar targets, Potentially Hazardous Asteroids (PHAs) and low- $\Delta V$ potential mission targets. Taxonomy was determined through a direct comparison with the 1341 main-belt and NearEarth objects in the SMASSII spectral survey (Bus and Binzel, 2002b). All P200 and the SMASSII reflectance spectra were normalized at $0.55 \mu \mathrm{m}$. Using the 49 channel spline-fit spectra in the SMASSII database, we defined a misfit by summing the deviations between the SMASSII reflectance values and the median of the P200 spectra within each SMASSII wavelength bin. The misfit between the P200 targets and the SMASSII objects were sorted and ranked. The P200 target was assigned the taxonomy of the bestfit SMASSII object. The main-belt asteroids in our sample were 


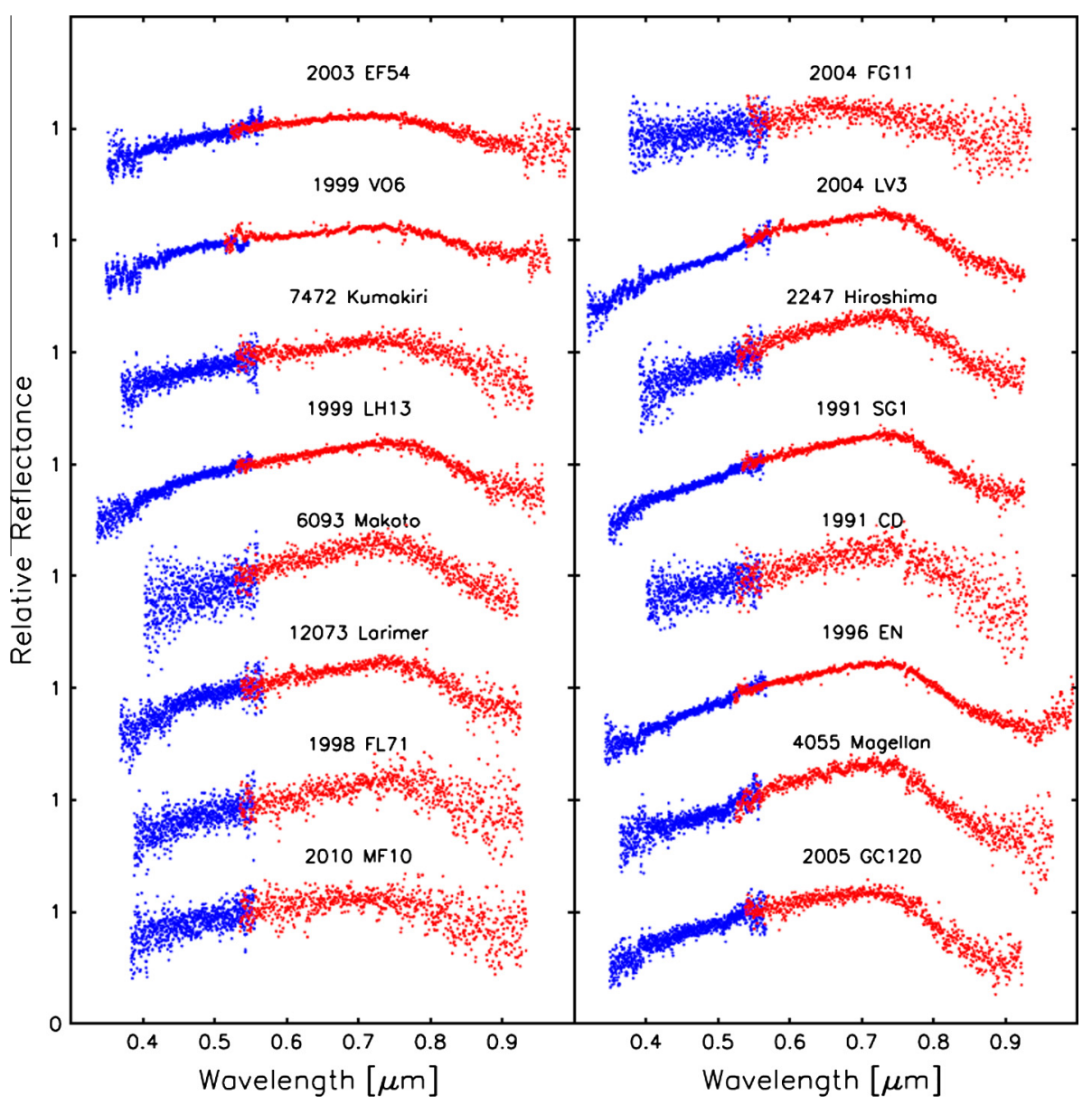

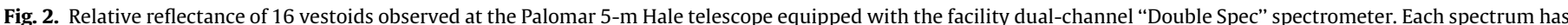

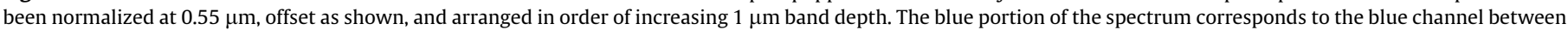

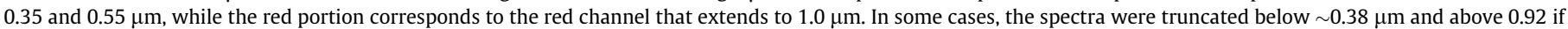
artifacts and noise in the data are dominant. (For interpretation of the references to color in this figure legend, the reader is referred to the web version of this article.)

identified by their SDSS colors and confirmed as V-type asteroids by our P200 spectroscopy. Two of the main-belt vestoids, 7472 Kumakiri and 1991 SG1, reside in the outer main belt well beyond the jovian 3:1 mean motion resonance near the Kirkwood Gap at $2.5 \mathrm{AU}$, as shown in Fig. 1. To have originated from 4 Vesta, these two objects would have had to successfully scatter across the $3: 1$, $5: 2$, and $7: 3$ resonances. It is possible that 7472 Kumakiri and 1991 SG1 are not derived from Vesta and represent crustal fragments of a disrupted basaltic parent body compositionally similar to 4 Vesta. Spectroscopic observations of 1459 Magnya, with a semi-major axis of $3.15 \mathrm{AU}$, revealed basaltic composition (Lazzaro et al., 2000).

\section{Broadband photometric observations: acquisition and reduction}

To maximize the solar phase coverage of our V-type NEO targets, an observational campaign of broad-band photometry was mounted across multiple sites: Table Mountain Observatory (Hicks, lead observer), Calar Alto Observatory (Duffard, lead observer), and Purple Mountain Observatory (Zhao, lead observer). The Table Mountain Observatory (TMO) is located 60 miles north-east of Pasadena, California at $2300 \mathrm{~m}$ elevation and features a $0.6-\mathrm{m}$ f/16 Ritchey-Chretien reflector equipped with CCD detector and Bessel BVRI filterset. TMO supports both on-site and remote observations. Fourteen nights of photometric data were acquired at TMO. The Calar Alto Astronomical Observatory (CA) is located at $2200 \mathrm{~m}$ elevation in the Sierra de Los Filabres Mountains of southern Spain. One night of R-band photometry at the CA 1.2-m reflector (CA1.2) and four nights of R-band photometry at the CA $2.2-\mathrm{m}$ reflector (CA2.2) were acquired. Two nights of photometric data were acquired with the Purple Mountain (PM) 1-m Xuyi Schmidt telescope. Table 4 lists the orbital elements and observational circumstances of our broad-band photometry targets. All data were reduced in a standard way by Hicks using IRAF and custom built code. Landolt standards (Landolt, 1992) were observed throughout each night at a wide range of airmass to provide photometric calibration. Landolt fields were observed at all three stations. The photometric accuracy of the PM and CA data was verified by reimaging the CA and PM fields at TMO and cross-calibrating background field stars, bringing the photometry to a self consistent filter system. Typical nightly calibration accuracies were $\sim 0.015$ mag for the $\mathrm{V}, \mathrm{R}$, and I filters and $\sim 0.022$ for the B filter data.

\section{Broadband photometric observations: results for individual objects}

In this section we discuss broadband observations of specific NEO targets, which have provided new rotation light curves and a composite solar phase curve; a new shape/spin model; BVRI colors; a new binary candidate; identification of new V-types NEOs; and a composite model to quantify solar phase reddening for V-type asteroids. 
Table 2

Measured spectral properties.

\begin{tabular}{|c|c|c|}
\hline & Spectral slope & Pseudo-band depth \\
\hline \multicolumn{3}{|l|}{ P200 vestoids } \\
\hline 1991 SG1 & $12.884 \pm 1.087$ & $0.330 \pm 0.014$ \\
\hline 1999 VO6 & $7.745 \pm 0.783$ & $0.152 \pm 0.008$ \\
\hline 2003 EF54 & $6.155 \pm 0.894$ & $0.136 \pm 0.008$ \\
\hline 2004 FG11 & $5.617 \pm 4.075$ & $0.287 \pm 0.037$ \\
\hline 2004 LV3 & $9.984 \pm 0.996$ & $0.320 \pm 0.012$ \\
\hline 2005 GC120 & $8.646 \pm 2.450$ & $0.444 \pm 0.017$ \\
\hline 2010 MF1 & $7.614 \pm 4.511$ & $0.267 \pm 0.030$ \\
\hline 2247 Hiroshima & $12.706 \pm 2.098$ & $0.330 \pm 0.012$ \\
\hline 4055 Magellan & $12.784 \pm 1.963$ & $0.396 \pm 0.015$ \\
\hline 6093 Makoto & $14.193 \pm 3.513$ & $0.229 \pm 0.023$ \\
\hline 7472 Kumakiri & $9.653 \pm 2.424$ & $0.213 \pm 0.017$ \\
\hline 8566 (1996 EN) & $11.826 \pm 0.960$ & $0.348 \pm 0.008$ \\
\hline 12073 Larimer & $9.303 \pm 2.031$ & $0.261 \pm 0.021$ \\
\hline 35965 (1999 LH13) & $10.998 \pm 1.106$ & $0.222 \pm 0.011$ \\
\hline 19165 (1991 CD) & $14.423 \pm 4.530$ & $0.343 \pm 0.037$ \\
\hline 199432 (1998 FL71) & $13.706 \pm 4.390$ & $0.262 \pm 0.042$ \\
\hline \multicolumn{3}{|l|}{ SMASS II vestoids } \\
\hline 4 Vesta & $5.138 \pm 0.202$ & $0.201 \pm 0.011$ \\
\hline 1929 Kollaa & $15.899 \pm 1.302$ & $0.281 \pm 0.012$ \\
\hline 2045 Peking & $11.729 \pm 1.822$ & $0.244 \pm 0.011$ \\
\hline 2468 Repin & $11.456 \pm 1.810$ & $0.279 \pm 0.012$ \\
\hline 2508 Alupka & $10.561 \pm 0.158$ & $0.218 \pm 0.014$ \\
\hline 2511 Patterson & $11.884 \pm 1.600$ & $0.271 \pm 0.012$ \\
\hline 2547 Hubie & $9.142 \pm 1.114$ & $0.208 \pm 0.010$ \\
\hline 2566 Kirghizia & $12.156 \pm 1.193$ & $0.285 \pm 0.011$ \\
\hline 2579 Spartacus & $15.343 \pm 6.508$ & $0.342 \pm 0.017$ \\
\hline 2640 Hallstrom & $10.233 \pm 0.996$ & $0.230 \pm 0.011$ \\
\hline 2653 Principia & $12.913 \pm 2.515$ & $0.313 \pm 0.016$ \\
\hline 2704 Julian Lowe & $9.922 \pm 2.137$ & $0.285 \pm 0.011$ \\
\hline 2763 Jeans & $11.924 \pm 0.417$ & $0.233 \pm 0.013$ \\
\hline 2795 Lepage & $14.571 \pm 2.529$ & $0.289 \pm 0.012$ \\
\hline 2851 Harbin & $16.861 \pm 0.806$ & $0.354 \pm 0.014$ \\
\hline 2912 Lapalma & $13.346 \pm 1.543$ & $0.353 \pm 0.012$ \\
\hline 3155 Lee & $14.097 \pm 3.589$ & $0.288 \pm 0.012$ \\
\hline 3265 Fletcher & $7.039 \pm 0.352$ & $0.156 \pm 0.010$ \\
\hline 3307 Athabasca & $14.232 \pm 1.292$ & $0.387 \pm 0.013$ \\
\hline 3498 Belton & $7.676 \pm 0.727$ & $0.175 \pm 0.013$ \\
\hline 3536 Scleicher & $9.368 \pm 0.877$ & $0.197 \pm 0.013$ \\
\hline 3782 Celle & $9.752 \pm 1.563$ & $0.236 \pm 0.012$ \\
\hline 3849 Incidentia & $9.500 \pm 1.572$ & $0.226 \pm 0.014$ \\
\hline 3850 Peltier & $11.480 \pm 1.220$ & $0.309 \pm 0.012$ \\
\hline 3900 Knezevic & $11.860 \pm 1.104$ & $0.312 \pm 0.011$ \\
\hline 4188 Kitezh & $9.640 \pm 1.019$ & $0.244 \pm 0.011$ \\
\hline 4215 Kamo & $10.872 \pm 1.938$ & $0.242 \pm 0.012$ \\
\hline 4311 Zguridi & $9.193 \pm 2.442$ & $0.190 \pm 0.010$ \\
\hline 4434 Nikulin & $9.537 \pm 1.161$ & $0.253 \pm 0.012$ \\
\hline 4796 Lewis & $9.337 \pm 2.294$ & $0.290 \pm 0.012$ \\
\hline 4900 Maymelou & $11.167 \pm 0.713$ & $0.247 \pm 0.011$ \\
\hline 4977 Rauthgundis & $11.142 \pm 1.525$ & $0.342 \pm 0.009$ \\
\hline 4993 Cossard & $9.727 \pm 2.846$ & $0.244 \pm 0.010$ \\
\hline 5240 Kwasan & $10.288 \pm 0.478$ & $0.252 \pm 0.014$ \\
\hline 5379 Abehiroshi & $8.253 \pm 4.730$ & $0.230 \pm 0.018$ \\
\hline
\end{tabular}

The near-Earth Asteroid 4688 (1980 WF) was discovered photographically by Charles Kowal on November 291980 (IAUC 3549). Infrared photometry ( $N$-band; $10 \mu \mathrm{m}$ ) acquired by Veeder et al. (1989) yielded an albedo of 0.18 and effective diameter of $0.6 \mathrm{~km}$. The spectral class assigned to this asteroid has evolved from SQ (Chapman et al., 1994) to V (Binzel et al., 2004). As a high solar-phase vestoid target, we observed $1980 \mathrm{WF}$ over the course of four partial nights at TMO and one partial night with the CA2.2 between January 21 and February 6 2011. We measured a new rotational period for 1980 WF: using standard Fourier techniques we determined a best-fit synodic period $P_{\text {syn }}=7.60 \pm 0.02 \mathrm{~h}$ with a relatively high lightcurve amplitude of $\sim 0.8$ mag. We measured an absolute magnitude in the Bessel R-band (effective wavelength $\lambda_{\text {eff }}=0.63 \mu \mathrm{m}$ ) of $H_{R}=19.18 \mathrm{mag}$, assuming a phase parameter $g=0.15$. Our $1980 \mathrm{WF}$ phased lightcurve is plotted in Fig. 4.
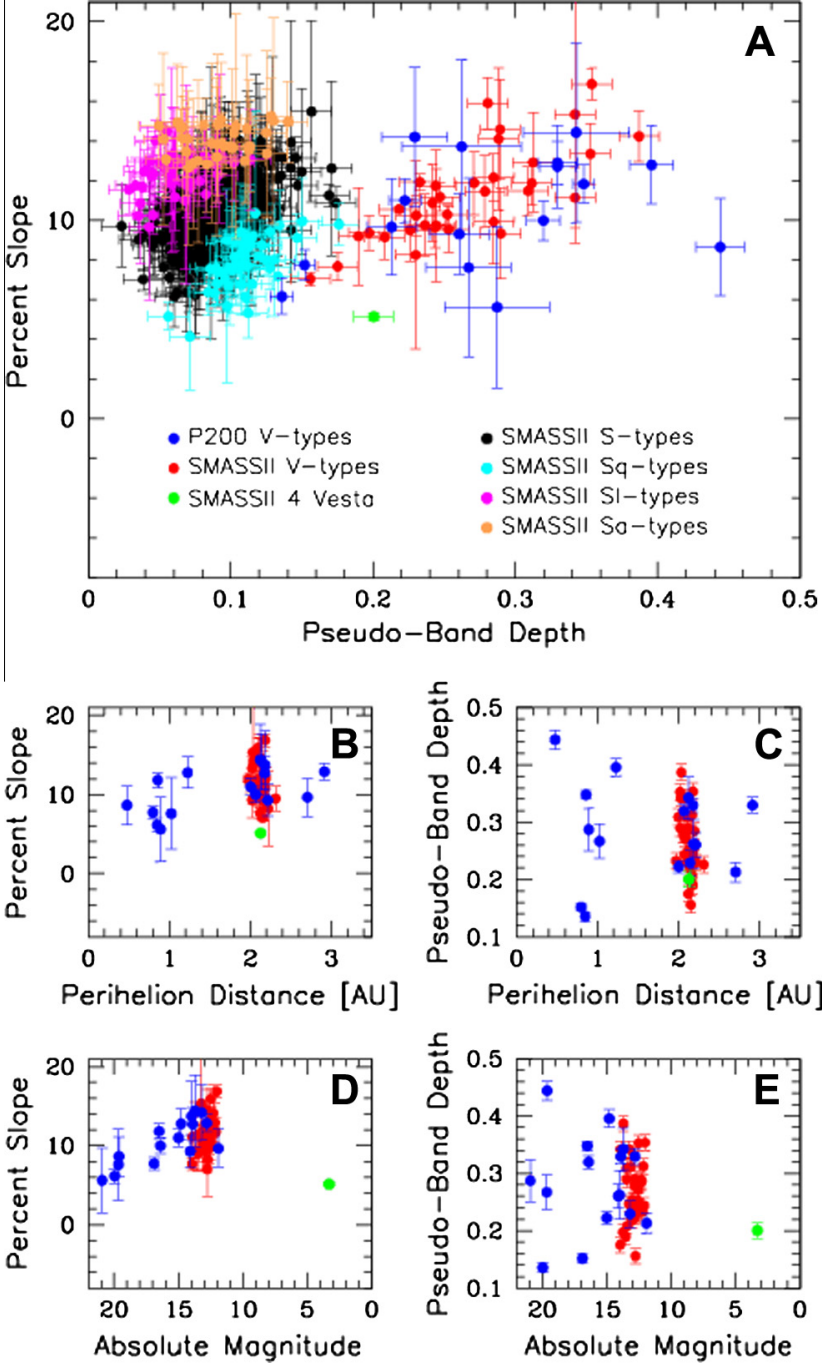

Fig. 3. Spectral slope and pseudo-band depth for P200 (blue) and SMASSII (red) vestoids. The slope is the percent change from 0.6 to $0.7 \mu \mathrm{m}$. The pseudo-band depth is the maximum reflectance wherever it exists in the spectra minus the reflectance at $0.85 \mu \mathrm{m}$ divided by the maximum reflectance. (No phase angle corrections have been applied.) There is no clear spectral difference between mainbelt and NEO vestoids, although Vesta appears to be an outlier. The bottom four panels show that there is no clear correlation between these slopes and band depths and perihelion distance or absolute magnitude, which is a proxy for size. (For interpretation of the references to color in this figure legend, the reader is referred to the web version of this article.)

Table 3

Linear correlations for measured P200 vestoid properties.

\begin{tabular}{llrr}
\hline$X$-axis & $Y$-axis & \multicolumn{1}{c}{$A$ (intercept) } & \multicolumn{1}{c}{$B$ (slope) } \\
\hline$q$ & $S$ & $6.865 \pm 1.455$ & $2.1902 \pm 0.7971$ \\
$q$ & $D$ & $0.297 \pm 0.053$ & $-0.0079 \pm 0.0288$ \\
$H$ & $S$ & $22.990 \pm 2.637$ & $-0.7878 \pm 0.1641$ \\
$H$ & $D$ & $0.289 \pm 0.125$ & $-0.0003 \pm 0.0078$ \\
$\alpha$ & $S$ & $12.292 \pm 1.167$ & $-0.0420 \pm 0.0227$ \\
$\alpha$ & $D$ & $0.276 \pm 0.038$ & $0.0002 \pm 0.0007$ \\
\hline
\end{tabular}

The near-Earth Asteroid 1981 Midas (1973 EA) was discovered by Charles Kowal (IAUC 2532). Muinonen et al. (2006) combined 6 nights of time-resolved photometry from the open literature and two additional nights of data from the object's 2004 apparition to search for a convex shape/pole solution using the optimum lightcurve inversion technique described by Kaasalainen et al. (2001). Although their dataset spanned 16 years, they were unable 
Table 4

Orbital elements and observing circumstances: Broad-band photometry targets.

\begin{tabular}{|c|c|c|c|c|c|c|c|c|}
\hline & UT date & Filter(s) & $r(\mathrm{AU})$ & $\Delta(\mathrm{AU})$ & $\alpha\left(^{\circ}\right)$ & $V($ mag $)$ & Tel. $^{\mathrm{a}}$ & Obs. $^{\text {b }}$ \\
\hline \multirow[t]{6}{*}{$4688(1980$ WF $)$} & & & & $(a, e, i, H=2.23,0.52,6,19)$ & & & & \\
\hline & 20110121.11 & $\mathrm{R}$ & 1.10 & 0.22 & 52.7 & 17.8 & TMO & $\mathrm{MH}$ \\
\hline & 20110122.15 & $\mathrm{R}$ & 1.10 & 0.22 & 52.3 & 17.8 & TMO & $\mathrm{MH}$ \\
\hline & 20110129.14 & $\mathrm{R}$ & 1.12 & 0.23 & 49.3 & 17.9 & TMO & $\mathrm{MH}$ \\
\hline & 20110204.15 & $\mathrm{R}$ & 1.14 & 0.25 & 46.6 & 18.0 & TMO & TB, MH \\
\hline & 20110205.86 & $\mathrm{R}$ & 1.15 & 0.26 & 45.9 & 18.1 & CA2.2 & $\mathrm{RD}$ \\
\hline \multirow[t]{5}{*}{1981 Midas } & & & & $(a, e, i, H=1.78,0.65,40,15.5)$ & & & & \\
\hline & 20110121.51 & BVRI & 1.26 & 0.86 & 51.0 & 17.6 & TMO & $\mathrm{MH}$ \\
\hline & 20110122.51 & BVRI & 1.25 & 0.84 & 51.6 & 17.5 & TMO & $\mathrm{MH}$ \\
\hline & 20110204.54 & BVRI & 1.11 & 0.65 & 61.6 & 17.0 & TMO & TB, MH \\
\hline & 20110212.55 & $\mathrm{R}$ & 1.02 & 0.56 & 70.3 & 16.8 & TMO & $\mathrm{MH}$ \\
\hline \multirow[t]{13}{*}{137052 Tjelvar (1998 V033) } & & & & $(a, e, i, H=1.25,0.89,15,16.844)$ & & & & \\
\hline & 20110115.16 & $\mathrm{R}$ & 1.48 & 0.59 & 25.2 & 17.7 & CA1.2 & $\mathrm{RD}$ \\
\hline & 20110121.30 & BVRI & 1.42 & 0.51 & 26.2 & 17.4 & TMO & $\mathrm{MH}$ \\
\hline & 20110122.34 & BVRI & 1.41 & 0.50 & 26.6 & 17.3 & TMO & $\mathrm{MH}$ \\
\hline & 20110129.32 & BVRI & 1.33 & 0.44 & 31.7 & 17.0 & TMO & $\mathrm{MH}$ \\
\hline & 20110204.28 & BVRI & 1.26 & 0.39 & 39.5 & 16.9 & TMO & $\mathrm{TB}, \mathrm{MH}$ \\
\hline & 20110204.88 & $\mathrm{R}$ & 1.25 & 0.39 & 40.5 & 16.9 & CA2.2 & $\mathrm{RD}$ \\
\hline & 20110211.24 & BVRI & 1.17 & 0.36 & 52.0 & 16.9 & TMO & $\mathrm{HR}, \mathrm{MH}$ \\
\hline & 20110212.24 & BVRI & 1.16 & 0.36 & 54.0 & 16.9 & TMO & $\mathrm{MH}$ \\
\hline & 20110213.26 & BVRI & 1.14 & 0.36 & 56.1 & 16.9 & TMO & $\mathrm{JF}$ \\
\hline & 20110220.61 & $\mathrm{R}$ & 1.04 & 0.36 & 72.2 & 17.2 & PM & $\mathrm{HZ}$ \\
\hline & 20110221.53 & $\mathrm{R}$ & 1.02 & 0.36 & 74.3 & 17.3 & PM & $\mathrm{HZ}$ \\
\hline & 20110225.17 & $\mathrm{R}$ & 0.97 & 0.36 & 82.4 & 17.5 & TMO & DM, MH \\
\hline \multirow[t]{8}{*}{253841 (2003 YG118) } & & & & $(a, e, i, H=2.28,0.64,8,17.051)$ & & & & \\
\hline & 20110129.45 & BVRI & 1.21 & 0.32 & 40.6 & 16.6 & TMO & $\mathrm{MH}$ \\
\hline & 20110204.44 & BVRI & 1.15 & 0.26 & 45.5 & 16.2 & TMO & $\mathrm{TB}, \mathrm{MH}$ \\
\hline & 20110205.17 & $\mathrm{R}$ & 1.14 & 0.25 & 46.6 & 16.1 & CA2.2 & $\mathrm{RD}$ \\
\hline & 20110206.21 & $\mathrm{R}$ & 1.13 & 0.24 & 47.9 & 16.1 & CA2.2 & $\mathrm{RD}$ \\
\hline & 20110207.15 & $\mathrm{R}$ & 1.13 & 0.24 & 49.0 & 16.0 & CA2.2 & $\mathrm{RD}$ \\
\hline & 20110211.43 & BVRI & 1.09 & 0.21 & 56.3 & 15.8 & TMO & $\mathrm{HR}, \mathrm{MH}$ \\
\hline & 20110212.44 & BVRI & 1.08 & 0.20 & 58.3 & 15.8 & TMO & $\mathrm{MH}$ \\
\hline \multirow[t]{6}{*}{4055 Magellan } & & & & $(a, e, i, H=1.82,0.33,23,14.8)$ & & & & \\
\hline & 20100809.17 & $\mathrm{R}$ & 1.26 & 0.58 & 52.4 & 16.0 & TMO & $\mathrm{MH}$ \\
\hline & 20100810.26 & $\mathrm{R}$ & 1.25 & 0.58 & 52.6 & 16.0 & TMO & TT \\
\hline & 20100812.26 & $\mathrm{R}$ & 1.25 & 0.58 & 52.8 & 16.0 & TMO & TT \\
\hline & 20100813.18 & $\mathrm{R}$ & 1.25 & 0.58 & 52.9 & 16.0 & TMO & TT \\
\hline & 20100814.18 & $\mathrm{R}$ & 1.25 & 0.57 & 53.0 & 16.0 & TMO & $\mathrm{MH}$ \\
\hline
\end{tabular}

a Telescope key: TMO = Table Mountain 0.6-m, CA2.2 = Calar Alto 2.2-m, CA1.2 = Calar Alto 1.2 m, PM = Purple Mountain 1-m Schmidt.

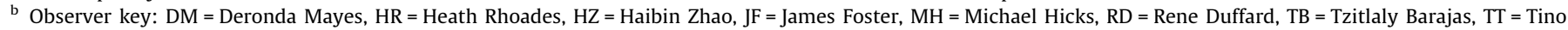
Truong.

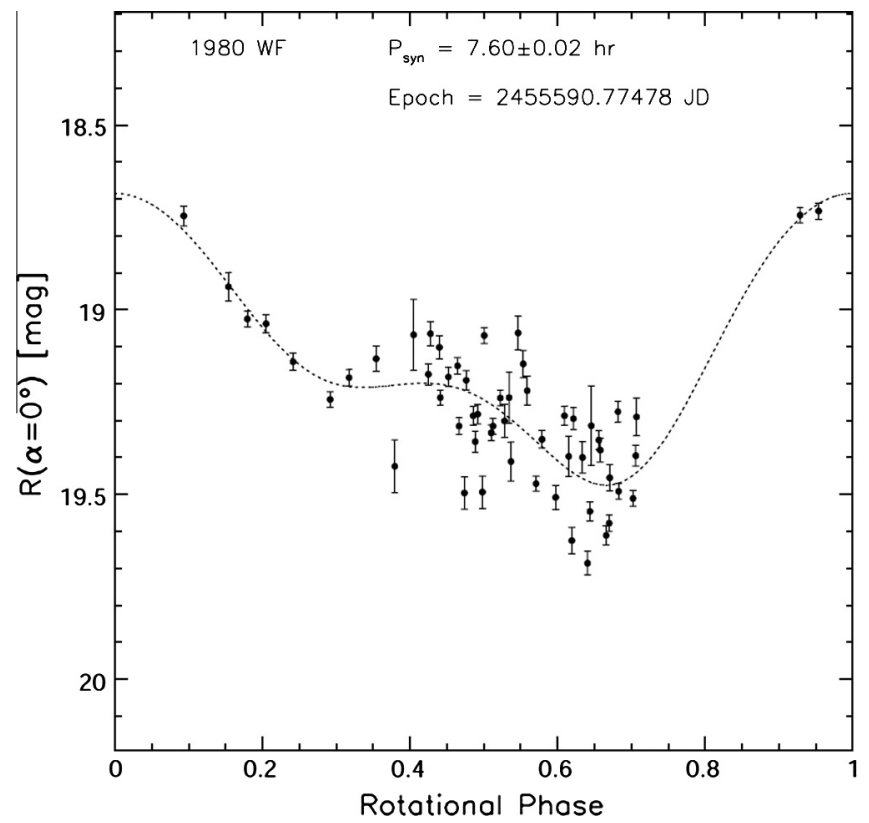

Fig. 4. Phased lightcurve for 4688 (1980 WF), which enables a tentative determination of the period of this vestoid, as well as its absolute magnitude. to determine a fixed pole/shape solution, but they did constrain the sidereal period $P_{\text {sid }}=5.22 \pm 0.04 \mathrm{~h}$. We obtained 3 nights of BVRI photometry and 1 night of R-band photometry of 1981 Midas at TMO, and found a best-fit synodic period $P_{\text {syn }}=5.314 \pm 0.002 \mathrm{~h}$ with a moderate lightcurve amplitude of $\sim 0.2 \mathrm{mag}$. Our photometry yielded an absolute magnitude $H_{R}=14.44 \mathrm{mag}$, assuming a phase parameter $g=0.15$. The phased 1981 Midas lightcurve is plotted in Fig. 5.

The near-Earth Asteroid 4055 Magellan (1985 DO2) was discovered photographically by Eleanor Helin. The object's taxonomic similarity with 4 Vesta at optical and near-IR wavelengths was first noted by Tholen et al. (1988) and was studied in depth spectroscopically by Whiteley (2001) and Binzel et al. (2004). Thermal observations by Delbó et al. (2003) determined a geometric albedo $\rho=0.31$, and, a rotational period of $7.475 \pm 0.001 \mathrm{~h}$ has been determined as part of Petr Pravec's Ondrejov Survey (http:// www.asu.cas.cz/ ppravec/neo.html). We obtained five partial nights of R-band photometry with the TMO 0.6-m telescope and found a best-fit synodic period $P_{\text {syn }}=7.491 \pm 0.005 \mathrm{~h}$ with a high lightcurve amplitude of $\sim 0.8$ mag. Our photometry yielded an absolute magnitude $H_{R}=14.56 \mathrm{mag}$, assuming a phase parameter $g=0.15$. Our phased 4055 Magellan lightcurve is plotted in Fig. 6 .

The near-Earth Asteroid 253841 (2003 YG118) was discovered by the LINEAR NEO Discovery Survey on December 17, 2003 (M.P.E.C. 2003-Y91). A rotatonal period $P=2.27 \pm 0.15$ h has been 


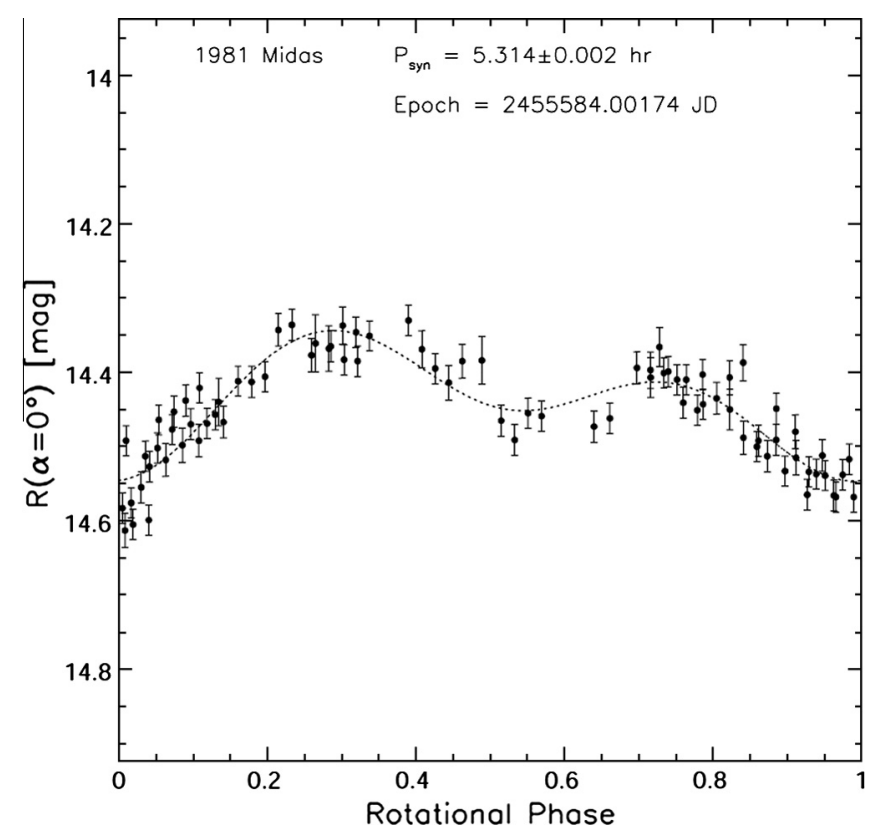

Fig. 5. Phased lightcurve for 1981 Midas, which led to an improved determination of its period (Kaasalainen et al., 2001) and a determination of its absolute magnitude.

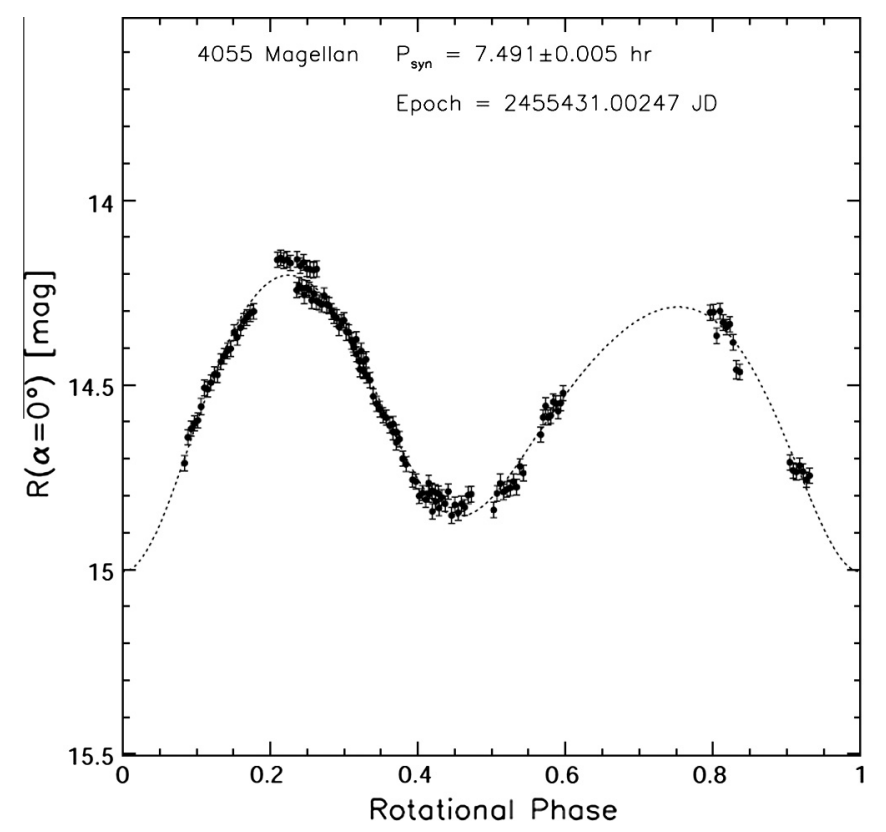

Fig. 6. Phased lightcurve for 4055 Magellan, showing a determination of its period. The slight offset at 0.2 phase is due to evolution of the illumination, projected area, and viewing geometries during the observing season.

reported by Brian Skiff (http://www.minorplanet.info/call.html). Visible and near-IR spectroscopy of this object was obtained by one of us (Duffard) at the 2.5-m Nordic Optical Telescope $(0.48-0.91 \mu \mathrm{m})$ and the 3.6-m Telescopio Nazionale Galileo (0.8$2.5 \mu \mathrm{m}$ ) in the object's discovery apparition (Duffard et al., 2006). The composite visible/near-IR reflectance spectrum of 2003 YG118 showed broad absorption features near 1 and $2 \mu \mathrm{m}$, suggestive of an admixture of mafic minerals. Using the method developed by Gaffey et al. (2002), which examines the 1 and $2 \mu \mathrm{m}$ band centers and band area ratios, Duffard et al. (2006) found 2003 YG118 to be comparable to 4 Vesta and that its spectrum could be modeled

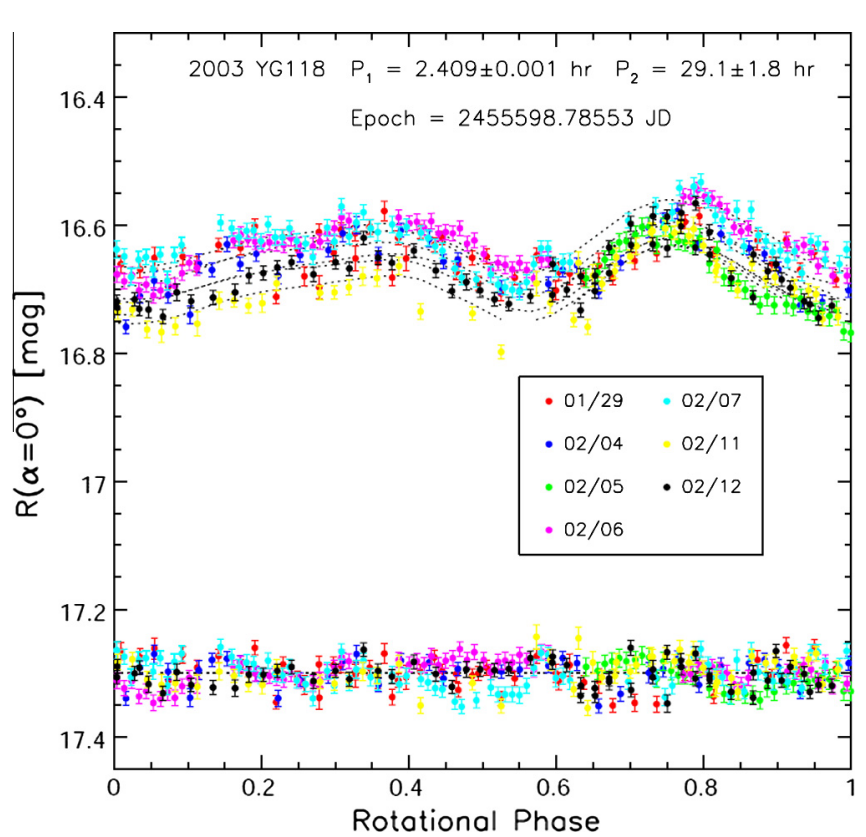

Fig. 7. Two-period lightcurve of 253841 (2003 YG118), identifying this asteroid as a binary. The seven nights of observation are shown with a model (dotted line) consisting of two additive components with noncommensurate periods. The bottom curve shows the residuals between the model and the data.

as a $85 / 15 \%$ mixture of low-calcium (othopryoxene) and highcalcium (clinopyroxene). Application of the Modified Gaussian Model (MGM) developed by Sunshine and Pieters (1993) by Duffard et al. (2006) also suggested a surface dominated by orthopyroxenes. 2003 YG118 was scheduled as a planetary radar target in February 2010, but equipment problems at the Arecibo facility unfortunately forced the cancellation of the radar observations (L. Benner, private communication). We observed 2003 YG118 over the course of 3 nights at optical wavelengths with the 2.2-m Calar Alto Telescope and 4 nights with the Table Mountain 0.6-m Telescope, as outlined in Table 4. Standard Fourier lightcurve analysis of our photometry initially pointed towards a low lightcurve amplitude and a rapid rotation period near $2.4 \mathrm{~h}$, near the rotational disruption rate for a strengthless spheroidal body (Margot et al., 2002). However, night-to-night variations in the reduced photometry suggested the lightcurve signature of a binary asteroid, as shown in Fig. 7. This result would not be surprising since approximately $17 \%$ of nearEarth asteroids larger than 200-m diameter are likely binary systems (Pravec et al., 2000, 2006). We modeled our observations using the double-period Fourier method as described by Pravec and Hahn (1997), which models the lightcurve as two additive components with noncommensurate periods. We found the bestfit solution $P_{1}=2.409 \pm 0.001 \mathrm{~h}$ and $P_{2}=29.1 \pm 1.8 \mathrm{~h}$ to be similar to other binary NEOs. We interpret the shorter period to be the rotation period of the primary and the longer period to reflect the orbital period of a tidally locked companion. Vesta-family binaries are not unknown: photometric observations of the main-belt vestoid 3782 Celle were found to be consistent with an eclipsing/ occulting binary system $P_{1}=3.84 \pm 0.01 \mathrm{~h}$ and $P_{2}=36.57 \pm 0.03 \mathrm{~h}$ (Ryan et al., 2004).

The near-Earth Asteroid 137052 Tjelvar (1998 VO33) was discovered by C.I. Lagerkvist at the La Silla 1-m Schmidt (M.P.E.C. 1998-W10) and spectral observations revealed a V-type taxonomy (Whiteley, 2001; Bus and Binzel, 2002b; Binzel et al., 2004). Krugly et al. (2002) reported an $8 \mathrm{~h}$ rotational period. We obtained 12 partial nights of rotationally resolved photometry of 137052 Tjelvar over a wide range of declinations and solar phase angles, including 1 night with the CA1.2, 1 night with the CA2.2, 2 nights with the 
PM Schmidt, and 8 nights at TMO. We determined a best-fit synodic period $P_{\text {syn }}=4.514 \pm 0.002 \mathrm{~h}$ with a relatively low lightcurve amplitude of $\sim 0.14 \mathrm{mag}$. We measured an absolute magnitude $H_{R}=16.60 \mathrm{mag}$, assuming a phase parameter $g=0.15$. The lightcurve of 137052 Tjelvar is plotted in Fig. 8.

Multi-filter rotationally resolved broad-band photometry can be used to explore compositional variability between objects and quantify wavelength-dependent solar phase effects. As listed in Table 5, BVRI observations for three of our NEO vestoids, 1981 Midas, 137052 Tjelvar, and 253841 (2003 YG118), were obtained over significant ranges of rotational and solar phase space. A standard color cycle consisted of seven exposures (R-I-R-V-R-B-R) observed multiple times allowing us to measure R-band lightcurves and instantaneous B-R, V-R, R-I colors. As with the optical spectroscopy, each object's mean colors were compared to the 1341 spectra archived in the SMASSII survey (Bus and Binzel, 2002a,b). Fig. 9 and Table 6 list the best-fit spectral analogs in the SMASSII dataset and our data confirms V-type taxonomy in each case. All asteroids flagged as V-type by their SDSS colors were confirmed as V-type by their P200 spectra. Aided by a wide range in observed solar phase angle, we used our nightly averaged colors to constrain wavelength dependent solar phase effects, as shown in Fig. 10 and Table 7. Our vestoid photometry suggests a subtle but statistically significant phase at BVRI wavelengths. The trends in B-R and R-I color imply that at high solar phase angle the optical spectrum is reddened and the $1-\mu \mathrm{m}$ pyroxene band is deeper, both of which have been seen in laboratory studies of HED meteorites (Reddy et al., 2012). If we extrapolate the mean BVRI colors back to $0^{\circ}$ phase, we find that the composite vestoid model matches 4 Vesta well, as shown in Fig. 11, suggesting that a significant fraction of observed variability at optical wavelengths may be due solely to phase angle effects. A similar study for S-type and Q-type asteroids found that about half of the effects of solar phase reddening could be attributed to viewing geometry (Sanchez et al., 2012).

As shown in Buratti and Veverka (1983), the disk integrated flux $\Phi(\alpha)$, which is normalized to unity at $\alpha=0^{\circ}$, can be converted to the surface phase function $f(\alpha)$, where $\alpha$ is the solar phase angle

$f(\alpha)=\Phi(\alpha) \cdot f_{0} /(1-\sin (\alpha / 2) \tan (\alpha / 2) \ln \cot (\alpha / 4))$

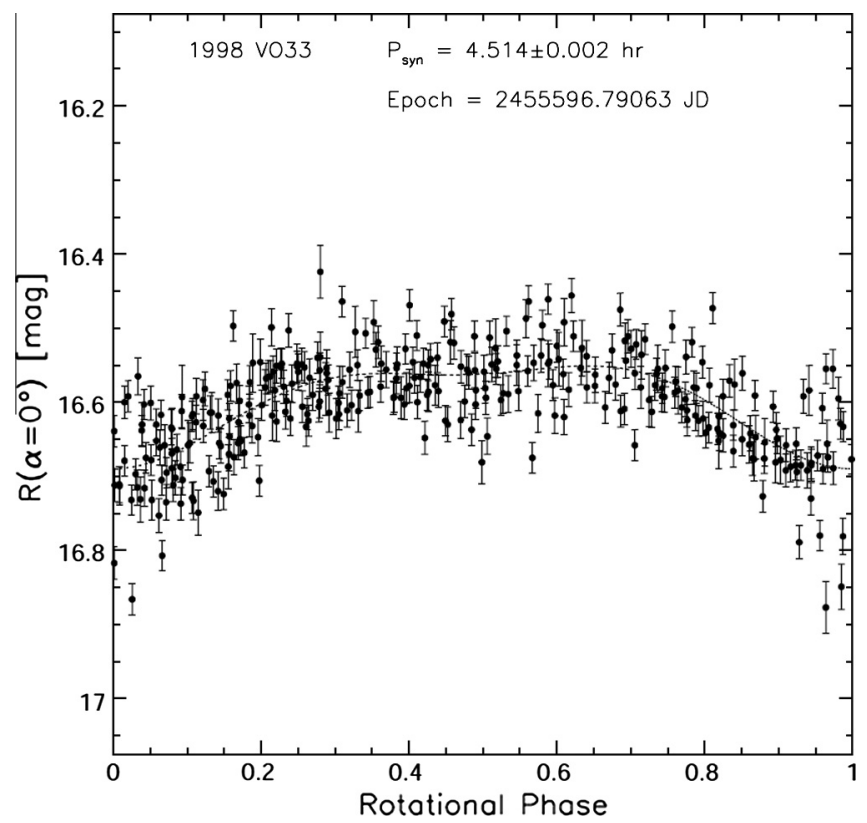

Fig. 8. Phased lightcurve of 137052 Tjelvar (1998 VO33), with a determination of its period. assuming a uniform sphere or knowledge of the local incident or emission angles and the Lommel-Seeliger scattering law. The solar phase function $f(\alpha)$ contains the effects of solar phase angle alone such as roughness and the single particle phase function. Knowledge of this function is necessary to obtain photometrically corrected spacecraft images. The changes in intensity due to the changing emission and incident angles are modeled in the trigometric term of Eq. (1). We restricted our photometry to objects with low lightcurve amplitude, allowing us to minimize shape effects. The upper panel of Fig. 12 illustrates our normalized solar phase curve in magnitude units. The dashed curve is a simple polynomial plus exponential term (to account for the opposition surge) fit of the form

$\Phi(\alpha)=A_{0} \exp \left(-A_{1} \alpha\right)+A_{2}+A_{3} \alpha+A_{4} \alpha^{2}+A_{5} \alpha^{3}+A_{6} \alpha^{4}$

After conversion from magnitude units to normalized linear units the $\Phi(\alpha)$ fit is applied to Eq. (1), which allowed us to generate a polynomial plus exponential fit to $f(\alpha)$ with $A_{0}=0.2255, A_{1}=$ $0.2300 \times 10^{-2}, \quad A_{2}=0.7745, \quad A_{3}=-1.6284 \times 10^{-2}, \quad A_{4}=1.2973 \times$ $10^{-4}, A_{5}=-0.4511 \times 10^{-6}, A_{6}=0.0556 \times 10^{-8}$. The bottom panel of Fig. 13 shows our empirical photometric model derived from our vestoid data, with the IAU HG model for comparison (see next section of more detail) Assuming Lommel-Seeliger scattering, our simple empirical model can be used to covert $I / F(\alpha)$ measured by the Dawn spacecraft to geometric albedo or to normalize the spacecraft data to any required illumination/viewing geometry.

\section{Broadband photometric observations: composite phase curve and photometric modeling}

One purpose of our investigation was to explore the photometric behavior of $\mathrm{V}$-type asteroids at solar phase angles inaccessible to ground based observers of 4 Vesta, which has a maximum excursion of $\sim 24^{\circ}$. The observed brightness of Near Earth vestoids at large solar phase angles $\left(\sim 80^{\circ}\right)$, which is more typical of the viewing geometries during the Dawn mapping period, enabled us to predict the intensity of the spacecraft images prior to orbit insertion. But in addition, our observations made it possible to construct a composite visible solar phase curve of V-type asteroids, similar to that published for S-type and C-type asteroids (Helfenstein and Veverka, 1989). This phase curve was fit to a physical photometric model and compared to the results of fits to other asteroid taxonomic types.

Over the past two and a half decades, photometric models describing the geophysical attributes of planetary surfaces have been published (Hapke, 1981, 1984, 1986, 1990; Buratti, 1985; Buratti and Veverka, 1985). The main parameters of these models are macroscopic roughness, which can be characterized by a mean slope angle (Hapke, 1984) or a mean depth-to-diameter ratio of a crater with specified surface coverage (Buratti and Veverka, 1985); the compaction state of the active upper regions of the surface (Irvine, 1966; Hapke, 1986); the single scattering albedo; and the directional scattering properties of the particles, which in turn are related to their size, shape, and composition. The models have been criticized in that they do not represent physical reality very well (see Shepard and Helfenstein, 2007, and Hakpe's response (Hapke, 2008; Hapke et al., 2009)) and that they are difficult to uniquely fit (Helfenstein et al., 1988). The first criticism can be mitigated somewhat by focusing on comparisons among the many fits that have been done over the years, and the second criticism can be largely avoided by gathering data over a full range of solar phase angles or by fitting both disk-integrated and disk-resolved measurements.

Following the techniques described in Hillier et al. (2011) we applied a Hapke model to a composite phase curve that contains 
Table 5

Broad-band colors.

\begin{tabular}{|c|c|c|c|c|c|}
\hline UT date & B-R (mag) & $\mathrm{V}-\mathrm{R}$ (mag) & R-I (mag) & Phase $\left(^{\circ}\right)$ & Number of cycles \\
\hline \multicolumn{6}{|l|}{1981 Midas } \\
\hline 20110121.51 & $1.305 \pm 0.056$ & $0.508 \pm 0.021$ & $0.138 \pm 0.023$ & 51.0 & 3 \\
\hline 20110122.51 & $1.382 \pm 0.033$ & $0.456 \pm 0.018$ & $0.181 \pm 0.011$ & 51.6 & 3 \\
\hline 20110204.54 & $1.380 \pm 0.032$ & $0.519 \pm 0.030$ & $0.179 \pm 0.033$ & 61.6 & 4 \\
\hline Mean colors & $1.359 \pm 0.049$ & $0.498 \pm 0.034$ & $0.167 \pm 0.030$ & & \\
\hline \multicolumn{6}{|c|}{137052 Tjelvar (1998 V033) } \\
\hline 20110121.30 & $1.233 \pm 0.015$ & $0.441 \pm 0.016$ & $0.215 \pm 0.015$ & 26.2 & 6 \\
\hline 20110122.34 & $1.144 \pm 0.177$ & $0.472 \pm 0.069$ & $0.219 \pm 0.025$ & 26.6 & 3 \\
\hline 20110129.32 & $1.230 \pm 0.019$ & $0.462 \pm 0.025$ & $0.215 \pm 0.011$ & 31.7 & 3 \\
\hline 20110204.28 & $1.218 \pm 0.059$ & $0.445 \pm 0.023$ & $0.255 \pm 0.018$ & 39.5 & 3 \\
\hline 20110211.24 & $1.309 \pm 0.084$ & $0.442 \pm 0.021$ & $0.179 \pm 0.043$ & 52.0 & 6 \\
\hline 20110212.24 & $1.302 \pm 0.077$ & $0.508 \pm 0.037$ & $0.224 \pm 0.050$ & 54.0 & 6 \\
\hline 20110213.26 & $1.370 \pm 0.062$ & $0.487 \pm 0.124$ & $0.192 \pm 0.066$ & 56.1 & 5 \\
\hline Mean colors & $1.280 \pm 0.086$ & $0.453 \pm 0.061$ & $0.212 \pm 0.050$ & & \\
\hline \multicolumn{6}{|c|}{253841 (2003 YG118) } \\
\hline 20110129.45 & $1.251 \pm 0.018$ & $0.452 \pm 0.036$ & $0.173 \pm 0.033$ & 40.6 & 8 \\
\hline 20110204.44 & $1.254 \pm 0.011$ & $0.336 \pm 0.012$ & $0.162 \pm 0.015$ & 45.5 & 6 \\
\hline 20110211.43 & $1.270 \pm 0.020$ & $0.436 \pm 0.011$ & $0.186 \pm 0.016$ & 56.3 & 6 \\
\hline 20110212.44 & $1.280 \pm 0.015$ & $0.443 \pm 0.014$ & $0.170 \pm 0.017$ & 58.3 & 6 \\
\hline Mean colors & $1.263 \pm 0.019$ & $0.445 \pm 0.022$ & $0.172 \pm 0.023$ & & \\
\hline
\end{tabular}

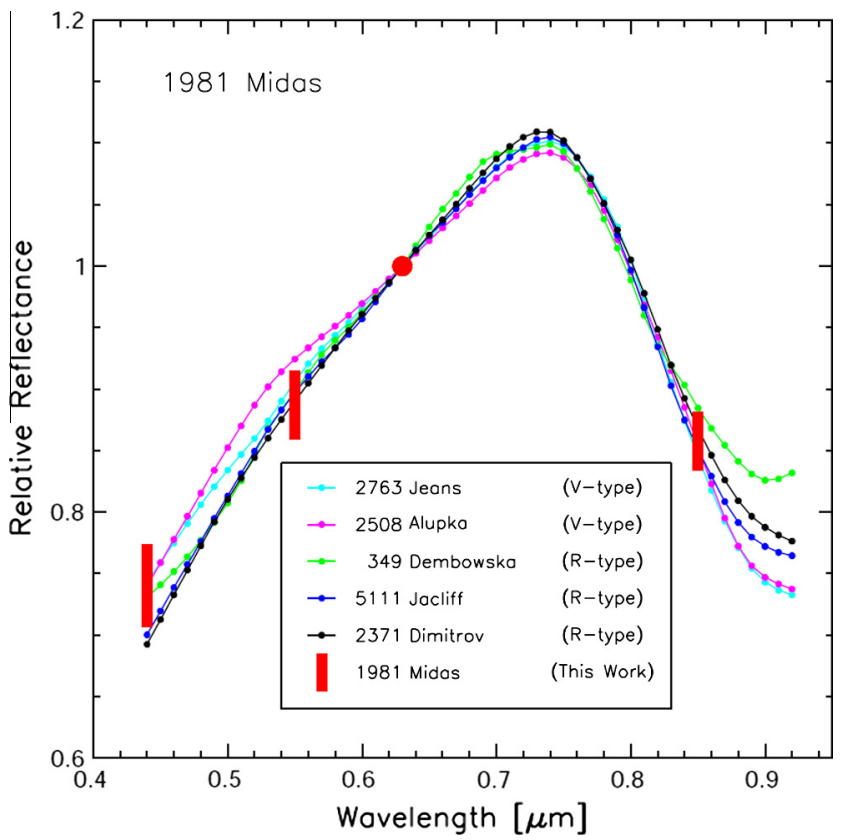

Fig. 9a. SMASSII BVRI colors (as measured from reflectance spectra) for 1981 Midas shown with spectra of the best fit spectral matches from the 1341 spectra in the data base (Bus and Binzel, 2002a,b). (For interpretation of the references to color in this figure legend, the reader is referred to the web version of this article.)

our observations of the near-Earth vestoids, the published observations of Vesta (Hasegawa et al., 2009; Reddy et al., 2012), Rosetta measurements (Fornasier et al., 2011) and the available full-disk observations of Vesta from Dawn (see Fig. 14). Disk-integrated measurements of Vesta's solar phase curve were extracted from the Framing Camera approach and survey data obtained in the Clear Filter. We selected radiometrically and geometrically calibrated images that included the full disk of Vesta: these data spanned the phase angle range from $29^{\circ}$ to $44^{\circ}$. The disk-integrated brightness of Vesta in each image was derived by performing aperture photometry, subtracting any residual background signal, and normalizing to a common spacecraft distance. The relative phase curve derived from Dawn was then normalized to the ground based observations as follows. First a fit was performed to Hapke's model

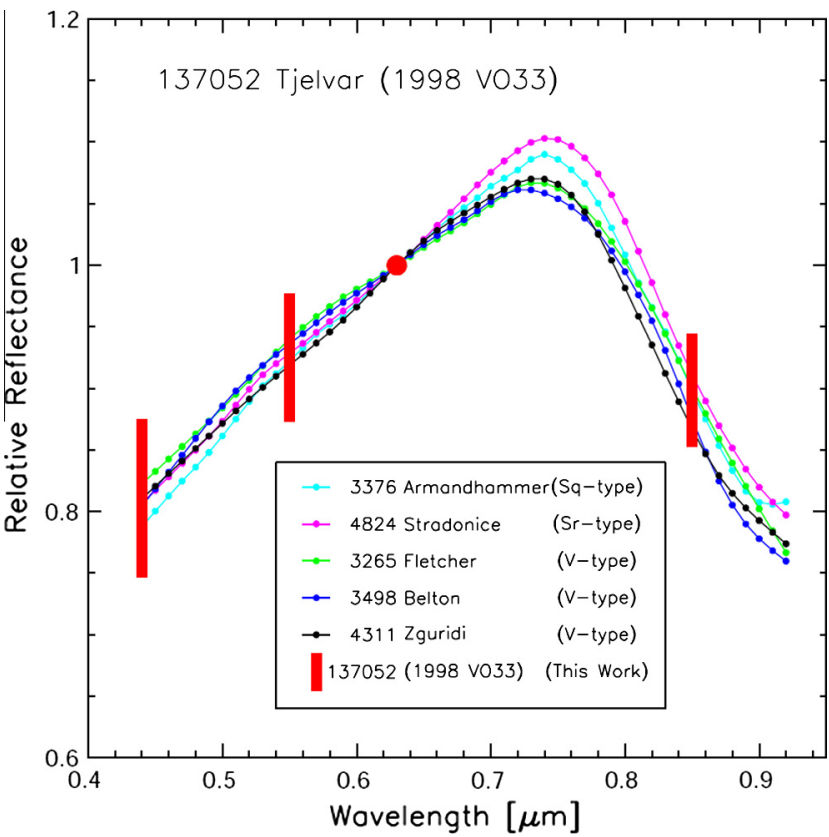

Fig. 9b. Same as Fig. 11a for 137052 Tjelvar (1998 V033).

excluding the Dawn data. A normalization factor needed to bring each Dawn data point in line with this fit was found. An average of these factors was then determined and applied to the Dawn data. For this fit, we characterize the macroscopic roughness by Hapke's mean slope parameter $(\theta)$ and the directional properties of the single particle phase function $P$ by the Henyey-Greenstein $g$,

$P(\cos \alpha, g)=\left(1-g^{2}\right) /\left(1+g^{2}+2 g \cos \alpha\right)^{3 / 2}$

where $g$ is the asymmetry parameter such that $g=1$ is purely forward scattering, $g=-1$ is purely backscattering, and $g=0$ is isotropic (Henyey and Greenstein, 1941). The single scattering albedo $\omega$ and the opposition surge parameters were also fit. For the opposition surge we fit the parameters $B_{0}$, which describes the amplitude of the surge, and $h$, which describes its width. The width depends on the particle sizes of regolith particles and how the particles are compacted: a small $h$ corresponds to a "fluffier" surface with more 


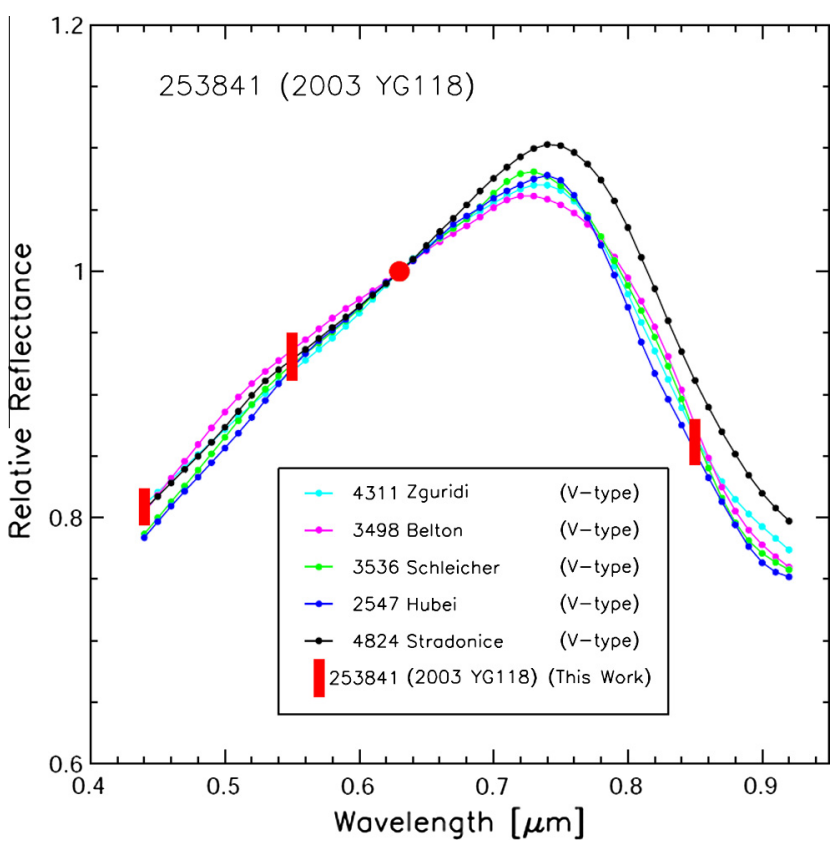

Fig. 9c. Same as Fig. 11a for 253841 (2003 YG118).

Table 6

Best-fit SMASSII matches.

\begin{tabular}{lll}
\hline Misfit & Object & Spectral type \\
\hline 1981 Midas & & \\
1.34 & 2763 Jeans & $\mathrm{V}$ \\
1.52 & 2508 Alupka & $\mathrm{V}$ \\
1.82 & 349 Dembowska & $\mathrm{R}$ \\
1.86 & 5111 Jacliff & $\mathrm{R}$ \\
2.08 & 2371 Dimitrov & $\mathrm{R}$ \\
2.08 & 4900 Maymelou & $\mathrm{V}$ \\
137052 Tjelvar(1998 V033) & & \\
0.46 & 3376 Armandhammer & $\mathrm{Sq}$ \\
0.55 & 4824 Stradonice & $\mathrm{Sr}$ \\
0.70 & 3265 Fletcher & $\mathrm{V}$ \\
0.74 & 3498 Belton & $\mathrm{V}$ \\
0.79 & 4311 Zguridi & $\mathrm{V}$ \\
1.00 & 3536 Schleicher & $\mathrm{V}$ \\
253841 (2003 YG118) & & \\
1.08 & 4311 Zguridi & $\mathrm{V}$ \\
1.31 & 3498 Belton & $\mathrm{V}$ \\
2.21 & 3536 Schleicher & $\mathrm{V}$ \\
2.68 & 2547 Hubei & $\mathrm{V}$ \\
2.93 & 4824 Stradonice & $\mathrm{Sr}$ \\
3.03 & 5240 Kwasan & $\mathrm{V}$ \\
\hline
\end{tabular}

void spaces between particles. We did not include the effects of coherent backscatter (Hapke, 1990) because our observations at small solar phase angles, particularly those $<1^{\circ}$, are not extensive enough to separately fit the opposition surge due to shadow hiding (Irvine, 1966) and the surge due to coherent backscatter. We find a backscattering coefficient, $g$, of -0.26 , a single scattering albedo of 0.51 , a mean slope roughness of $31.5^{\circ}$, and shadowing hiding opposition surge parameters $h$ and $B_{0}$ of 0.098 and 1.0, respectively.

Table 8 summarizes our results for roughness and the single scattering albedo along with previous work on small bodies. Fig. 14 shows a phase curve that includes all the vestoid and Vesta data along with the Hapke model fit and the IAU model fit from Fig. 13. The vestoid measurements have been corrected for rotational effects, and the Dawn observations have been corrected for both rotational effects and projection effects due to the nonsphericity of the asteroid.
The phase curve shown in Fig. 14 can be used to compute the phase integral, which expresses the directional scattering properties of a planetary body:

$q=2 \int_{0}^{\pi} \Phi(\alpha) \sin (\alpha) d \alpha$

The Hapke model fit yields a phase integral of $0.44 \pm 0.02$. With knowledge of the integral scattering properties of the V-asteroids over a large range of solar phase angles, and a measure of the brightness at $0^{\circ}$, which in turn yields a geometric albedo, the Bond albedo can be estimated. The Bond albedo $\left(A_{B}\right)$ is the ratio of the integrated flux reflected by the satellite to the integrated flux received, and it is equal to the geometric albedo (p) times the phase integral. (The bolometric Bond albedo is this quantity integrated over all wavelengths, but we do not have the spectral coverage to determine this parameter.) Both disk integrated and disk-resolved values of the bolometric Bond albedo are fundamental parameters for understanding energy balance and volatile transport on a planetary body. Using the visible geometric albedo of $0.34 \pm 0.02$ from the JPL Horizons data base, we find a Bond albedo of $0.15 \pm 0.03$.

Our R-band photometry allowed us to explore solar phase behavior of vestoids at high solar phase angles. Fig. 13 illustrates the disk integrated photometry for four vestoids and 4 Vesta obtained by our team. The $H-G$ magnitude describes the reduced magnitude of an atmosphereless small body as a function of absolute magnitude $H$ and solar phase parameter $G$ and was adopted by the IAU in 1985 (Bowell et al., 1989). The vestoid solar phase behavior is reasonably well described by a range of phase parameter $0.28<G<0.34$, although the $H-G$ model begins to deviate from the observed behavior at solar phase angle $\alpha>60^{\circ}$, more so than the Hapke model (see also Fig. 14). The relatively high $G$ is consistent with a bright, high albedo asteroid where multiple scattering is not insignificant.

\section{Spectral observations: disk-integrated spectra of Vesta over a complete rotation}

In addition to our study of NEO vestoids, we obtained spectra of 4 Vesta over a full rotation curve near the time of Dawn orbit insertion. The purpose of these observations was to provide a standard set of well-calibrated spectral data to serve as calibration sets for the Dawn instruments and to obtain rotational phase variations of Vesta's south polar regions, which had not been imaged by HST (Zellner et al., 1997; Li et al., 2010), and which are a focus of Dawn's mapping mission. We obtained two nights of medium-resolution disk integrated spectroscopy of the asteroid at the Hale 200-in. telescope on July 7 and 8, 2011, at sub-Earth latitudes of $\sim 40^{\circ}$ south, closely matching the Dawn approach trajectory. Table 9 lists observing circumstances. Over 600 spectral images of the asteroid were acquired, unfortunately through strong and variable cirrus. The brightness of Vesta $(V \sim 6 \mathrm{mag})$ forced us to use the instrument's most narrow slit of 0.5 arcsec, and to defocus Vesta to well over 10 arcsec in diameter (the standard stars were not defocused). These factors may have introduced some small scale artifacts into our data. Data analysis procedures were the same as that described for the vestoid objects, except in this case we observed the standard stars with a 0.5 arcsec slit.

The top of Fig. 15 shows a composite spectrum of all our observations. Each observation was ratioed to the solar analogue star, normalized to $0.5 \mu \mathrm{m}$ and median filtered as a group. In addition, we investigated hemispheric differences in the spectra. The bottom panel shows the median average of all spectra with sub-observer longitudes between $100^{\circ}$ and $280^{\circ}$ (IAU convention) ratioed to the averaged spectrum (red) and all other longitudes (green). There 

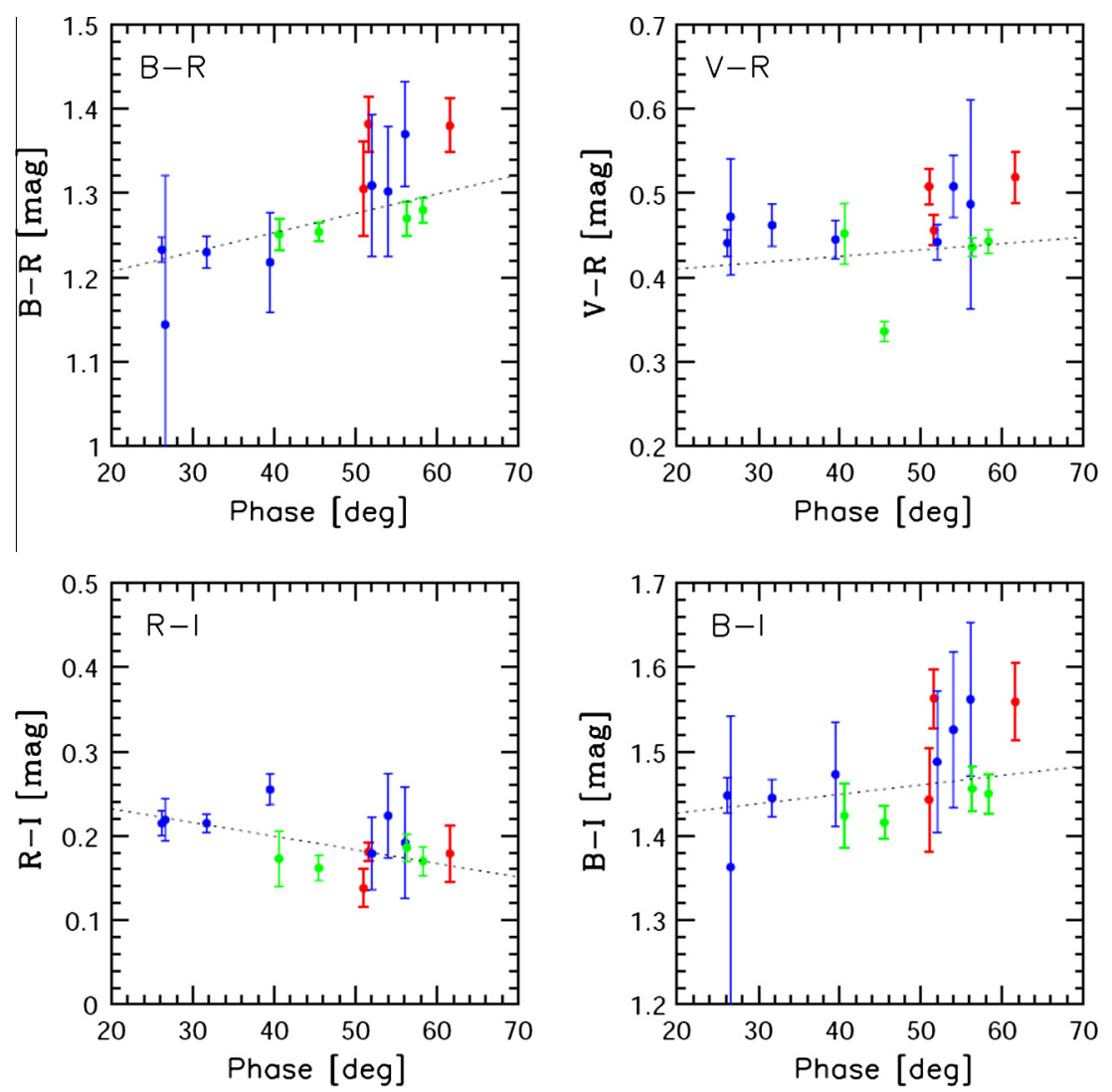

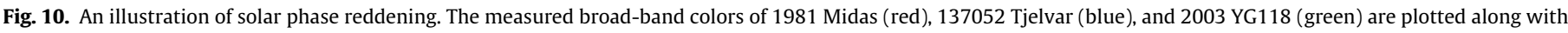

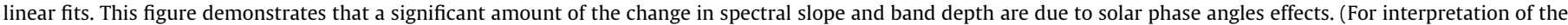
references to color in this figure legend, the reader is referred to the web version of this article.)

Table 7

Vestoid phase reddening.

\begin{tabular}{llc}
\hline & Intercept $(\mathrm{mag})$ & Slope $\left(\times 10^{-3}\right)\left(\mathrm{mag} \mathrm{deg}^{-1}\right)$ \\
\hline B-R & $1.161 \pm 0.024$ & $2.301 \pm 0.526$ \\
V-R & $0.395 \pm 0.025$ & $0.749 \pm 0.497$ \\
R-I & $0.264 \pm 0.019$ & $-1.608 \pm 0.427$ \\
B-I & $1.405 \pm 0.033$ & $1.122 \pm 0.722$ \\
\hline
\end{tabular}

is a substantial difference in the visible slope between the two hemispheres. (We are not confident in the differences shown at $0.73,0.82$, and $0.95 \mu \mathrm{m}$, as they are likely artifacts due to night sky emissions or telescope flexure). The band depth centered near $0.95 \mu \mathrm{m}$ does not appear to change as a function of longitude.

One of the most valuable analyses of these spectra is a comparison of the spectral properties of the south pole of Vesta with the equatorial regions. Ground-based observations of the visible and near-IR reflectance of Vesta have long suggested that the body is differentiated (McCord et al., 1970), with the confirmation by the Dawn Mission (Russell et al., 2012). Previous spectra of Vesta were obtained at more equatorial latitudes (Fig. 16), although Gaffey (1997) hypothesized that a small, shallow impact basin at the south pole of Vesta was more diogenitic than the average surface. A large body of petrologic evidence suggests that the HEDs may come from different depths of 4 Vesta (see McSween et al., 2011, for a review). The diogenite meteorites are assumed to originate deep in Vesta's crust, while the eucrites come from lava flows onto its surface or from shallow dikes and plutons. Howardites are believed to be a mixture of the two. The large crater at Vesta's south pole (Schenk et al., 2012; Russell et al., 2012) appears to have excavated a large portion of the crust of Vesta, exposing mineral types

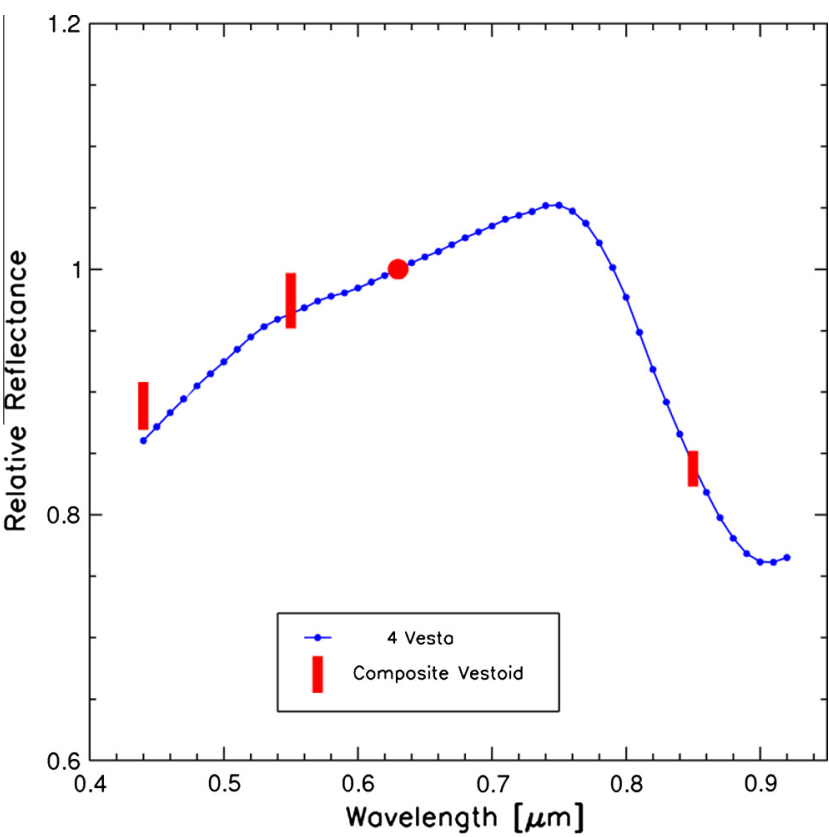

Fig. 11. Composite vestoid color model reduced to $0^{\circ}$ solar phase angle. This figure shows that the phase-corrected model spectrum of our composite set of V-type asteroids fits closely to the SMASSII spectrum of 4 Vesta. (For interpretation of the references to color in this figure legend, the reader is referred to the web version of this article.)

from deeper in the crust and mantle of the asteroid. If indeed this excavated portion of Vesta is enriched in diogenitic material, it 


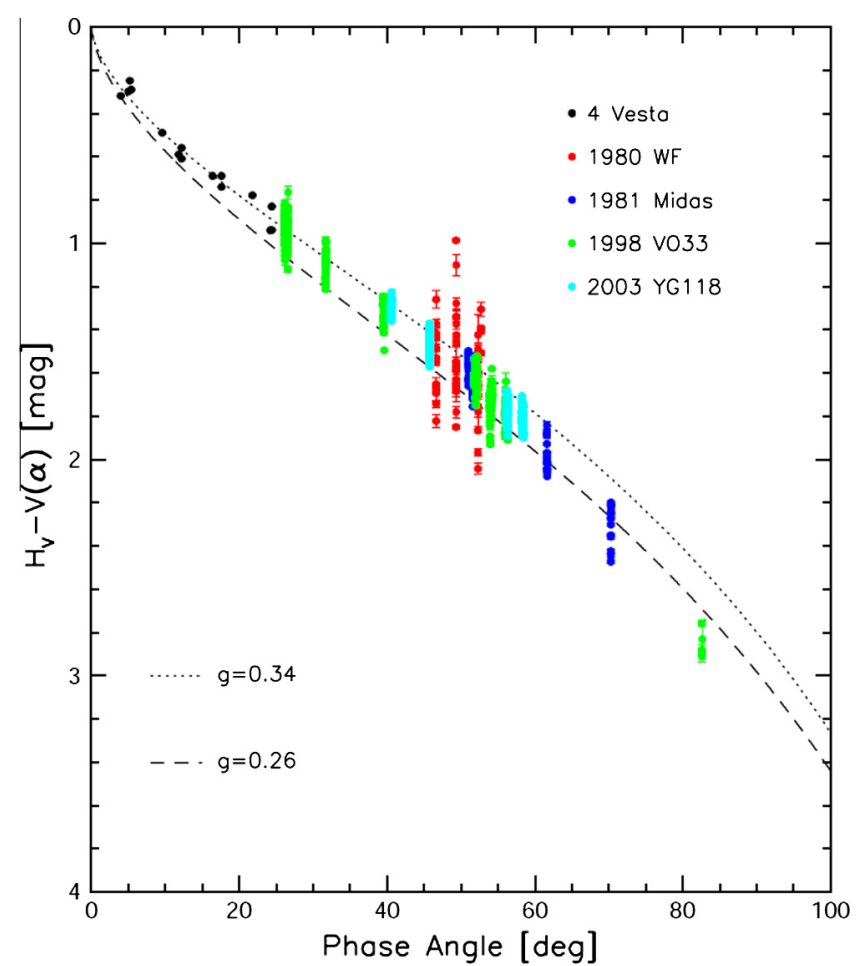

Fig. 12. A composite solar phase curve for the vestoids we observed and the 4 Vesta data from Reddy et al. (2012) and our observations. Two fits to the IAU model parameter $g$, which depends on the shape of the phase curve, are shown. Neither predicts the brightness of vestoids at large solar phase angles. More sophisticated photometric modeling, shown in Fig. 13, is needed to explain the decrease in brightness beyond $50^{\circ}$.

should show up in our spectrum of the south pole. Fig. 16 shows our composite spectrum, the SMASS spectrum of Vesta which is more representative of Vesta's equatorial region, and spectra of typical diogenite and eucrite meteorites at various particle sizes, as archived in the Relab data base (http://www.planetary. brown.edu/relab). Although our spectrum closely resembles the eucrites shortward of $0.7 \mu \mathrm{m}$, it is more similar to diogenites than the more equatorial SMASS spectrum, which suggests the polar regions are enriched in diogenitic material lower in the crust or core of Vesta. Similarly the band at $0.95 \mu \mathrm{m}$ is deeper than the SMASS spectrum, which is also more characteristic of a diogenitic composition. In addition this band position is closer to that of the diogenite samples, although given the noise in our spectrum at the critical wavelengths, this result is only suggestive. First results from the Dawn Visible and Infrared Spectrometer (VIR) also suggest that the south pole is more diogenitic, based on an analysis of the $2 \mu \mathrm{m}$ band (De Sanctis et al., 2012). Previous groundbased work on vestoids shows specific correlations between 1929 Kollaa and a more eucritic composition similar to 4 Vesta's crust (Kelley et al., 2003).

\section{Discussion, comparisons with other small bodies, and conclusions}

Our photometric investigation of V-type asteroids yielded a number of important results that are complementary to the investigation being performed by the Dawn Spacecraft at 4 Vesta. First, we find that the smaller vestoids exhibit deeper $1 \mu \mathrm{m}$ absorption bands and bluer slopes in the visible portion of the spectrum. With the classic view of space weathering, which entails the reddening of the visible slope and dampening of absorption band strength (Pieters et al., 2000; Hapke, 2001) this result implies that they
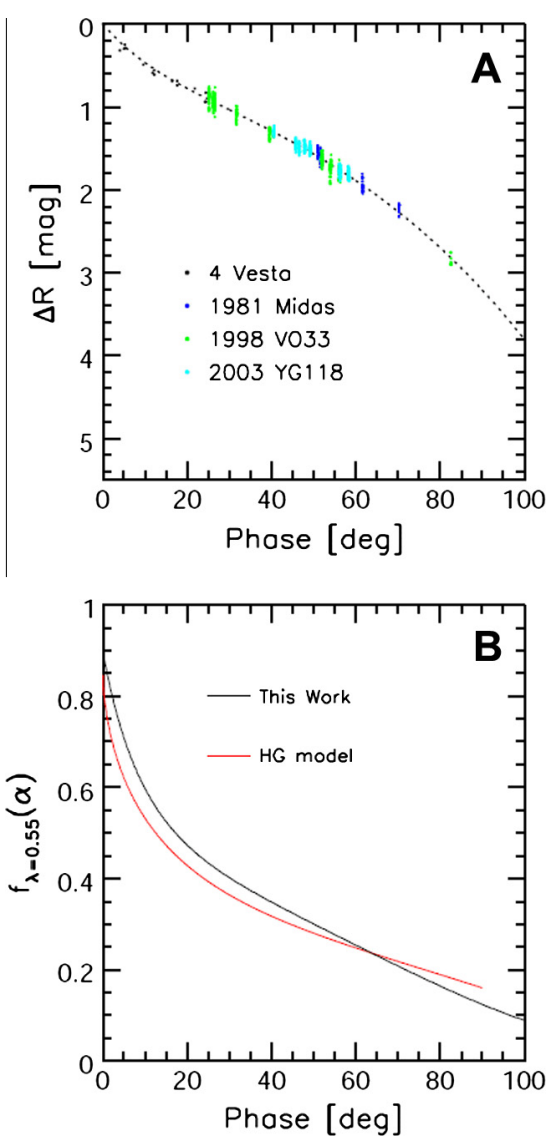

Fig. 13. (Top) Our empirical photometric model derived from a composite of the vestoid data. (Bottom) The fit to the data (blue line) compared to the IAU model, which cannot predict the brightness at large solar phase angles. (For interpretation of the references to colour in this figure legend, the reader is referred to the web version of this article.)

are younger and were injected into the inner Solar System more recently. Binzel et al. (2004) found this trend with S-type asteroids. However, with the finding that space weathering on Vesta works in a different way than that on S-type asteroids, with more exposed surfaces tending to be brighter and bluer due to communication of particles (McCord et al., 2012; Pieters et al., 2012), this connection is less clear. Laboratory ion bombardment experiments suggest that the V-type asteroids should be redder than HEDs (Vernazza et al., 2006; Fulvio et al., 2012). We confirmed two vestoids, 7472 Kumakiri and 1991 SG1, beyond the Jupiter 3:1 resonance. Either there is an unknown mechanism for moving these objects out that far, or there is another source body for V-type asteroids. Finally, we find that phase reddening may explain much of the spectral diversity in vestoids, and perhaps other asteroids if solar phase color corrections are done properly.

Our results show that the particles comprising the surface of 4 Vesta, and vestoids in general, are back-scattering, similar to the surfaces of all other airless bodies in the Solar System. The directional scattering properties are most similar to S-type asteroids. Although we do not have extensive measurements of the opposition surge of Vesta, especially at very small phase angles, which would enable us to determine if coherent backscatter is present, the amplitude and width of the phase curve is typical of other small bodies, and the amount of compaction in the upper regolith is similar to S-type asteroids. Where Vesta does appear to be unique is in its roughness: its mean slope angle of $31.5^{\circ}$ is similar to that of objects such as the saturnian satellite Phoebe (Buratti et al., 2008), which is in a highly collisional environment (Bottke 


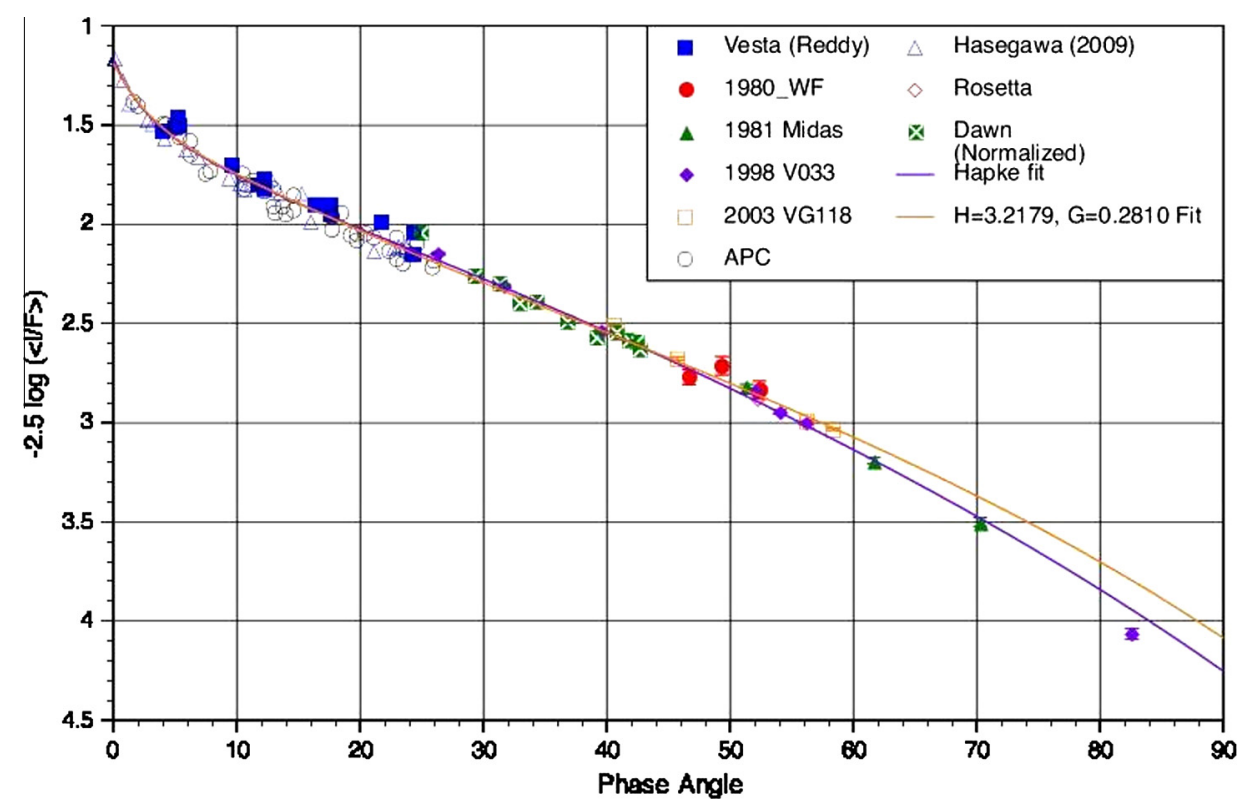

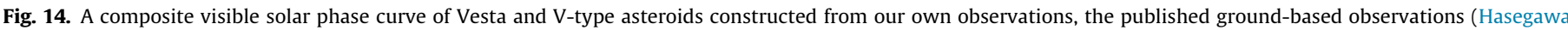

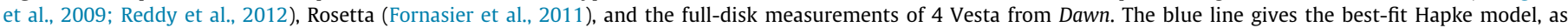

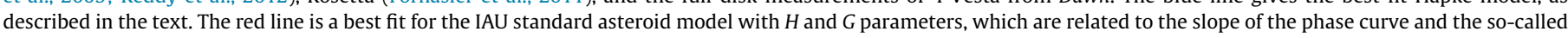
opposition surge at phase angles below $5^{\circ}$. (For interpretation of the references to color in this figure legend, the reader is referred to the web version of this article.)

Table 8

The roughness and single scattering albedo of selected small bodies.

\begin{tabular}{|c|c|c|c|}
\hline Object & Single scattering albedo & Slope angle $\left({ }^{\circ}\right)$ & Reference \\
\hline Vestoids + Vesta & 0.510 & 32 & This work \\
\hline Average C-type & 0.037 & 20 & Helfenstein and Veverka (1989) \\
\hline Average $\mathrm{S}$ & 0.23 & 20 & Helfenstein and Veverka (1989) \\
\hline Phoebe & 0.07 & 32 & Buratti et al. (2008) \\
\hline \multirow[t]{2}{*}{ 19/P Borrelly } & 0.020 & 20 & Buratti et al. (2004) \\
\hline & 0.057 & $\sim 35$ & Li et al. (2007) \\
\hline Moon & 0.25 & 20 & Buratti (1985) \\
\hline
\end{tabular}

Table 9

4 Vesta spectroscopy observing circumstances.

\begin{tabular}{|c|c|c|c|c|c|c|c|c|c|c|}
\hline \multirow[t]{3}{*}{ UT date } & \multirow[t]{3}{*}{$r(\mathrm{AU})$} & \multirow[t]{3}{*}{$\Delta(\mathrm{AU})$} & \multirow[t]{3}{*}{$\alpha\left(^{\circ}\right)$} & \multicolumn{2}{|c|}{ UT time } & \multirow[t]{3}{*}{ Num. exp. } & \multicolumn{4}{|c|}{ Sub-observer } \\
\hline & & & & \multirow[t]{2}{*}{ Start } & \multirow[t]{2}{*}{ Stop } & & \multicolumn{2}{|l|}{ Start } & \multicolumn{2}{|l|}{ Stop } \\
\hline & & & & & & & Long. $\left({ }^{\circ}\right)$ & Lat. $\left(^{\circ}\right)$ & Long. $\left({ }^{\circ}\right)$ & Lat. $\left(^{\circ}\right)$ \\
\hline July 7, 2011 & 2.22 & 1.30 & 14.8 & $09: 27$ & $11: 39$ & 121 & 121.0 & -38.6 & 269.3 & -38.6 \\
\hline July 8, 2011 & 2.22 & 1.30 & 14.5 & $08: 28$ & $12: 20$ & 504 & 232.3 & -38.5 & 132.9 & -38.5 \\
\hline
\end{tabular}

et al., 2010). Vesta may have had an unusually violent collisional history that was perhaps associated with the creation of the V-type asteroids. Our result supports recent radar findings that achondritic igneous rocky asteroids - the $\mathrm{E}$ and $\mathrm{V}$ types - have the largest surface roughness (Benner et al., 2008). Benner et al. suggest that this high surface roughness may be due to unusual mechanical properties or mineralogy of V-type and E-type asteroids. An additional factor may be the more substantial regolith that Vesta has, in comparison to smaller asteroids with a lower surface gravity. These regolith particles could serve as a supply to form rough aggregates of particles. Roughness models are scale-invariant: they include features from craters, mountains, and faults, down to clumps of particles below the resolution limit of the camera. When combined with radar measurements, our results show that the $\mathrm{V}$-class is rough at both small scales (sub-millimeter through centemeter) and the decimeter scales to which radar is sensitive. For the Moon, Helfenstein and Shepard (1999) showed that roughness is dominated by features at the small end of the scale. Whatever the cause of the higher macroscopic roughness of vestoids, our result suggests Vesta and its family have had a more violent collisional history, comparable to that of the saturnian satellite Phoebe, which has been battered by the family of outer irregular saturnian satellites (Buratti et al., 2008; Bottke et al., 2010).

Work by Binzel et al. (2004) suggests that space weathering is important in the NEO population. However, the similarity in visible spectral slope and the band depth at $0.85 \mu \mathrm{m}$ between the Main Belt and Near-Earth vestoids (Fig. 3) suggests that much if not most of the space weathering can be explained by spectral reddening with solar phase angle that has not been fully corrected for. Finally, space weathering on Vesta does not appear to entail the classic effects of space weathering on S-type bodies, viz., reddening of the visible spectral slope and muting of spectral band depths; on the contrary slight spectral bluing and brightening appears to occur (McCord et al., 2012; Pieters et al., 2012). The effect may 

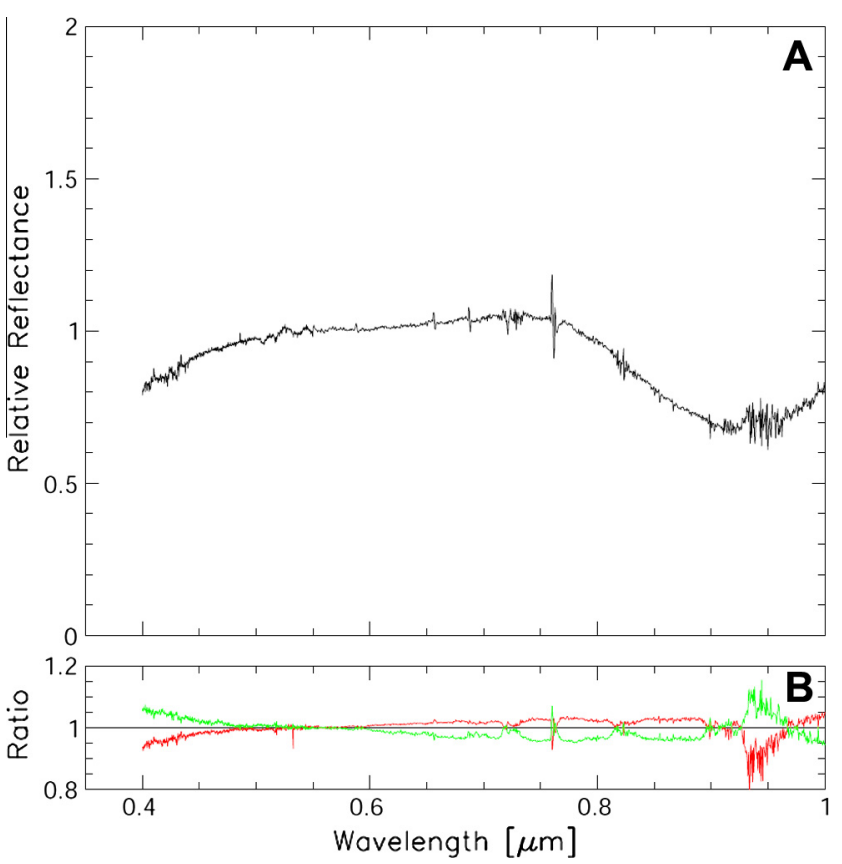

Fig. 15. The top graph shows the longitudinally averaged Vesta spectrum obtained in July 2011. The bottom graph shows the spectra binned into two ranges of longitude: $100-280^{\circ}$ (red) and all other longitudes (green), with the former data set exhibiting a redder slope towards the blue end of the spectrum. (For interpretation of the references to color in this figure legend, the reader is referred to the web version of this article.)

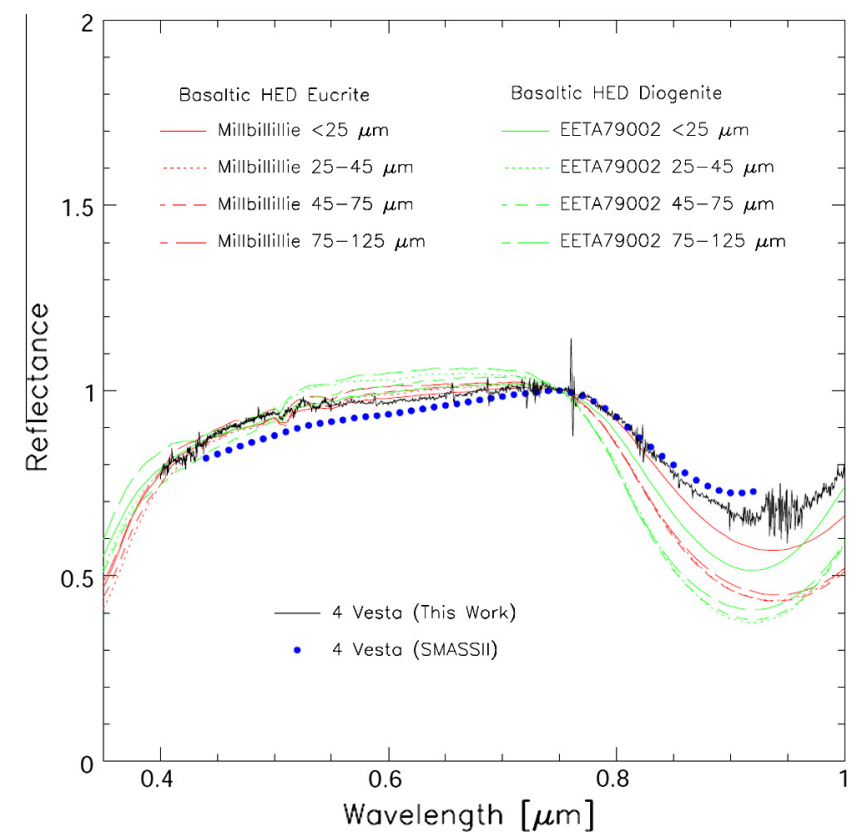

Fig. 16. Our composite spectrum of Vesta compared with the SMASS spectrum that represents more equatorial viewing geometries. Also shown are spectra of typical Diogenite and Eucrite meteorites consisting of several particle sizes, from the Relab data base (http://www.planetary.brown.edu/relab). Although our spectrum closely matches that of the eucrites, it is more similar to diogenitic meteorites than the SMASS spectrum, which suggests the south polar regions may be more diogenitic in composition.

be entirely absent on smaller NEOs as the "Vesta-like" space weathering is due to the pulverization of surface particles. These grains may simply leave the smaller NEOs.

\section{Acknowledgments}

This research was carried out at the Jet Propulsion Laboratory, California Institute of Technology under contract to the National Aeronautics and Space Administration. We acknowledge support from the Dawn Participating Scientist program and the NASA Near Earth Objects Observation (NEO-O) Program. Funding for the creation and distribution of the SDSS Archive has been provided by the Alfred P. Sloan Foundation, the Participating Institutions, the National Aeronautics and Space Administration, the National Science Foundation, the U.S. Department of Energy, the Japanese Monbukagakusho, and the Max Planck Society. The SDSS Web site is http://www.sdss.org/.

The SDSS is managed by the Astrophysical Research Consortium (ARC) for the Participating Institutions. The Participating Institutions are The University of Chicago, Fermilab, the Institute for Advanced Study, the Japan Participation Group, The Johns Hopkins University, the Korean Scientist Group, Los Alamos National Laboratory, the Max-Planck-Institute for Astronomy (MPIA), the Max-Planck-Institute for Astrophysics (MPA), New Mexico State University, University of Pittsburgh, University of Portsmouth, Princeton University, the United States Naval Observatory, and the University of Washington. This work was in part based on observations collected at the Centro Astronómico Hispano Alemán (CAHA) at Calar Alto, operated jointly by the Max-Planck Institut für Astronomie and the Instituto de Astrofísica de Andalucía (CSIC).

This research utilizes spectra acquired by the NASA Relab facility at Brown University. We are very grateful to the reviewers of this manuscript.

\section{References}

Benner, L. et al., 2008. Near-Earth asteroid surface roughness depends on compositional class. Icarus 198, 294-304.

Binzel, R.P., Rivkin, A.S., Stuart, J.S., Harris, A.W., Bus, S.J., Burbine, T.H., 2004. Observed spectral properties of Near-Earth Objects: Results for population distribution, source regions, and space weathering processes. Icarus 170, 259294.

Binzel, R.P. et al., 2010. Earth encounters as the origin of fresh surfaces on nearEarth asteroids. Nature 463, 331-334.

Bottke, W., Nesvorný, D., Vokrouhlický, Morbidelli, A., 2010. The irregular satellites: The most collisionally evolved populations in the Solar System. Astron. J. 139, 994-1014

Bowell, E., Hapke, B., Domingue, D., Lumme, K., Peltoniemi, J., Harris, A.W., 1989. Application of photometric models to asteroids. In: Asteroids II. Proceedings of the Conference, Tucson, AZ, March 8-11, 1988, Tucson, AZ. University of Arizona Press, Tucson, pp. 524-556.

Buratti, B.J., 1985. Application of a radiative transfer model to bright icy satellites. Icarus 61, 208-217.

Buratti, B.J., Veverka, J., 1983. Voyager photometry of Europa. Icarus 55, 93-110.

Buratti, B.J., Veverka, J., 1985. Photometry of rough planetary surfaces: The role of multiple scattering. Icarus $64,320-328$.

Buratti, B.J. et al., 2004. 9969 Braille: Deep Space 1 infrared spectroscopy, geometric albedo, and classification. Icarus 167, 129-135.

Buratti, B.J. et al., 2008. Infrared $(0.83-5.1 \mu \mathrm{m})$ photometry of Phoebe from the Cassini Visual Infrared Mapping Spectrometer. Icarus 193, 309-322.

Burbine, T.H., Duffard, R., Buchanan, P.C., Cloutis, E.A., Binzel, R.P., 2011. Spectroscopy of O-type asteroids. Lunar Planet. Sci. 42.

Bus, S.J., Binzel, R.P., 2002a. Phase II of the small main-belt asteroid spectroscopic survey: The observations. Icarus 158, 106-145.

Bus, S.J., Binzel, R.P., 2002b. Phase II of the small main-belt asteroid spectroscopic survey: A feature-based taxonomy. Icarus 158, 146-177.

Chapman, C.R., Harris, A.W., Binzel, R., 1994. Physical properties of near-Earth asteroids: Implications for the hazards issue. In: Gehrels, T. (Ed.), Hazards Due to Asteroids and Comets. University of Arizona Press, Tucson, pp. 537-549.

Cruikshank, D.P., Tholen, D.J., Bell, J.F., Hartmann, W.K., Brown, R.H., 1991. Three basaltic Earth-approaching asteroids and the source of the basaltic meteorites. Icarus 89, 1-13.

De Sanctis, M.C. et al., 2012. Spectroscopic characterization of mineralogy and its diversity across Vesta. Science 336, 697-700.

Delbó, M., Harris, A.W., Binzel, R.P., Pravec, P., Davies, J.K., 2003. Keck observations of near-Earth asteroids in the thermal infrared. Icarus 166, 116-130.

Duffard, R., de León, J., Licandro, J., Lazzaro, D., Serra-Ricart, M., 2006. Basaltic asteroids in the Near-Earth Objects population: A mineralogical analysis. Astron. Astrophys. 456, 775-781. 
Fornasier, S., Mottola, S., Barucci, M.A., Sierks, H., Hviid, S., 2011. Photometric observations of Asteroid 4 Vesta by the OSIRIS cameras onboard the Rosetta spacecraft. Astron. Astrophys. 533, L9-L13.

Fulvio, D., Brunetto, R., Vernazza, P., Strazzulla, G., 2012. Space weathering of Vesta and V-type asteroids: New irradiation experiments on HED meteorites. Astron. Astrophys. 537, L11-L16.

Gaffey, M.J., 1997. Surface lithologic heterogeneity of Asteroid 4 Vesta. Icarus 127, $130-157$.

Gaffey, M.J., Cloutis, E.A., Kelley, M.S., Reed, K.L., 2002. Mineralogy of asteroids. In: Bottke, W.F., Cellino, A., Paolicchi, P., Binzel, R.P. (Eds.), Asteroids III. University of Arizona Press, Tucson, pp. 183-234.

Hapke, B., 1981. Bidirectional reflectance spectroscopy. 1: Theory. J. Geophys. Res. 86, 3039-3054.

Hapke, B., 1984. Bidirectional reflectance spectroscopy. 3: Correction for macroscopic roughness. Icarus 59, 41-59.

Hapke, B., 1986. Bidirectional reflectance spectroscopy. 4: The extinction coefficient and the opposition effect. Icarus 67, 264-280.

Hapke, B., 1990. Coherent backscatter and the radar characteristics of outer planet satellites. Icarus 88, 407-417.

Hapke, B., 2001. Space weathering from Mercury to the asteroid belt. J. Geophys. Res. 106, 10039-10074.

Hapke, B., 2008. Bidirectional reflectance spectroscopy. Icarus 195, 918-926.

Hapke, B.W., Shepard, M.K., Nelson, R.M., Smythe, W.D., Piatek, J.L., 2009. A quantitative test of the ability of models based on the equation of radiative transfer to predict the bidirectional reflectance of a well-characterized medium. Icarus 199, 210-218.

Hasegawa, S. et al., 2009. BRz' phase function of Asteroid 4 Vesta during the 2006 opposition. Lunar Planet. Sci. 40. \#1503.

Helfenstein, P., Shepard, M.K., 1999. Submillimeter-scale topography of the lunar regolith. Icarus $141,107-131$.

Helfenstein, P., Veverka, J., 1989. Physical characterization of asteroid surfaces from photometric analysis. In: Binzel, R., Gehrels, T., Matthews, M.S. (Eds.), Asteroids II. University of Arizona Press, Tucson, pp. 557-593.

Helfenstein, P., Veverka, J., Thomas, P.C., 1988. Uranus satellites: Hapke parameters from Voyager disk-integrated photometry. Icarus 74, 231-239.

Henyey, L.G., Greenstein, J., 1941. Diffuse radiation in the Galaxy. Astrophys. J. 93, 70-83.

Hicks, M.D., Buratti, B.J., 2004. The spectral variability of Triton from 1997-2000. Icarus 171, 210-218.

Hillier, J.K., Bauer, J.M., Buratti, B.J., 2011. Photometric modeling of Asteroid 5535 Annefrank from Stardust observations. Icarus 211, 546-552.

Irvine, W., 1966. The shadowing effect in diffuse reflection. J. Geophys. Res. 71, 2931-2941.

Ivezic, Z., Juric, M., Lupton, R.H., Tabachnik, S., Quinn, T., and the SDSS Collaboration, 2002. Survey and other telescope technologies and discoveries. In: Tyson, J.A., Wolff, S. (Eds.), Proceedings of SPIE, vol. 4836, pp. 98-103.

Kaasalainen, M., Torppa, J., Muinonen, K., 2001. Optimization methods for asteroid lightcurve inversion. II. The complete inverse problem. Icarus 153, 37-51.

Kelley, M.S., Vilas, F., Gaffey, M.J., Abell, P.A., 2003. Quantified mineralogical evidence for a common origin of 1929 Kollaa with 4 Vesta and the HED meteorites. Icarus 167, 215-218.

Krugly, Y.N., Belskaya, I.N., Shevchenko, V.G., Chiorny, V.G., Velichko, F.P., Mottola, S., Erikson, A., Hahn, G., Nathues, A., Neukum, G., Gaftonyuk, N.M., Dotto, E., 2002. The Near-Earth Objects follow-up program. IV. CCD photometry in 19961999. Icarus 158, 294-304.

Landolt, A.U., 1992. UBVRI photometric standard stars in the magnitude range 11.516.0 around the celestial equator. Astron. J. 104, 340-371.

Lazzaro, D. et al., 2000. Discovery of a basaltic asteroid in the outer main belt. Science 288, 2033-2035.

Li, Y.-J., A'Hearn, M.F., McFadden, L.A., Belton, M.J.S., 2007. Photometric analysis and disk-resolved thermal modeling of Comet 19P/Borrelly from Deep Space 1 data. Icarus 188, 195-211.

Li, Y.-J., McFadden, L.A., Thomas, P.C., Mutchler, M.J., Parker, J.W., Young, E.F., Russell, C.T., Sykes, M.V., Schmidt, B.E., 2010. Photometric mapping of Asteroid 4 Vesta's southern hemisphere with Hubble Space Telescope. Icarus 208, 238251.

Marchi, S., Paolicchi, P., Lazzarin, M., Magrin, S., 2006. A general spectral slopeexposure relation for S-type main belt and near-Earth asteroids. Astron. J. 131, $1138-1141$.

Marchi, S.De., Sanctis, M.C., Lazzarin, M., Magrin, S., 2010. On the puzzle of space weathering alteration of basaltic asteroids. Astrophys. J. 721, L172-L176.
Margot, J.L. et al., 2002. Binary asteroids in the near-Earth object population. Science 296, 1145-1148.

McCord, T.B., Adams, J.B., Johnson, T.V., 1970. Asteroid Vesta: Spectral reflectivity and compositional implications. Science 168, 1445-1447.

McCord, T.B. et al., 2012. Dark material on Vesta from the infall of carbonaceous volatile-rich material. Nature 491, 83-86.

McSween, H.Y., Mittlefehldt, D.W., Beck, A.W., Mayne, R.G., McCoy, T.J., 2011. HED meteorites and their relationship to the geology of Vesta and the Dawn Mission. Space Sci. Rev. 163, 141-174.

Moskovitz, N.A., Jedicke, R., Gaidos, E., Willman, M., Nesvorný, D., Fevig, R., Ivezić, Ž., 2008. The distribution of basaltic asteroids in the Main Belt. Icarus 198, 77-90.

Muinonen, K. et al., 2006. In: Valsecchi, G.B., Vokrouhlický, D., Milani, A. (Eds.), Near Earth Objects, Our Celestial Neighbors: Opportunity and Risk. Cambridge University Press, England, pp. 309-320.

Nesvorný, D., Jedicke, R., Whiteley, R.J., Ivezić, Ž., 2005. Evidence for asteroid space weathering from the Sloan Digital Sky Survey. Icarus 173, 132-152.

Oke, J.B., Gunn, J.E., 1982. An efficient low resolution and moderate resolution spectrograph for the Hale Telescope. Publ. Astron. Soc. Pacific 94, 586-594.

Pieters, C.M. et al., 2000. Space weathering on airless bodies: Resolving a mystery with lunar samples. Meteorit. Planet. Sci. 35, 1101-1107.

Pieters, C.M. et al., 2012. Vesta's distinctive space weathering captures regolith mixing processes. Nature 491, 79-82.

Pravec, P., Hahn, G., 1997. Two-period lightcurve of 1994 AW1: Indication of a binary asteroid? Icarus 127, 431-440.

Pravec, P., Šarounová, L., Rabinowitz, D.L., Hicks, M.D., Wolf, M., Krugly, Y.N., Velichko, F.P., Shevchenko, V.G., Chiorny, V.G., Gaftonyuk, N.M., Genevier, G., 2000. Two-period lightcurves of 1996 FG3, 1998 PG, and (5407) 1992 AX: One probable and two possible binary asteroids. Icarus 146, 190-203.

Pravec, P., Scheirich, P., Kušnirák, P., Šarounová, L., Mottola, S., Hahn, G., Brown, P., Esquerdo, G., Kaiser, N., Krzeminski, Z., Pray, D.P., Warner, B.D., Harris, A.W., Nolan, M.C., Howell, E.S., Benner, L.A.M., Margot, J.-L., Galád, A., Holliday, W., Hicks, M.D., Krugly, Yu.N., Tholen, D., Whiteley, R., Marchis, F., Degraff, D.R., Grauer, A., Larson, S., Velichko, F.P., Cooney, W.R., Stephens, R., Zhu, J., Kirsch, K. Dyvig, R., Snyder, L., Reddy, V., Moore, S., Gajdoš, Š., Világi, J., Masi, G., Higgins, D., Funkhouser, G., Knight, B., Slivan, S., Behrend, R., Grenon, M., Burki, G., Roy, R., Demeautis, C., Matter, D., Waelchli, N., Revaz, Y., Klotz, A., Rieugné, M., Thierry, P., Cotrez, V., Brunetto, L., Kober, G., 2006. Photometric survey of binary near-Earth asteroids. Icarus 181, 63-93.

Reddy, V. et al., 2012. Photometric, spectral phase and temperature effects on 4 Vesta and HED meteorites: Implications for the Dawn Mission. Icarus 217, 153168.

Roig, F., Gil-Hutton, R., 2006. Selecting candidate V-type asteroids from the analysis of the Sloan Digital Sky Survey colors. Icarus 183 (2), 411-419.

Russell, C.T., Raymond, C.A., Coradini, A., McSween, H.Y., Zuber, M.T., Nathues, A., De Sanctis, M.C., Jaumann, R., Konopliv, A.S., Preusker, F., Asmar, S.W., Park, R.S., Gaskell, R., Keller, H.U., Mottola, S., Roatsch, T., Scully, J.E.C., Smith, D.E. Tricarico, P., Toplis, M.J., Christensen, U.R., Feldman, W.C., Lawrence, D.J., McCoy, T.J., Prettyman, T.H., Reedy, R.C., Sykes, M.E., Titus, T.N., 2012. Dawn at Vesta: Testing the protoplanetary paradigm. Science 336 (6082), 684-686.

Ryan, W.H., Ryan, E.V., Martinez, C.T., 2004. 3782 Celle: Discovery of a binary system within the Vesta family of asteroids. Planet. Space Sci. 52, 1093-1101.

Sanchez, J.A., Reddy, V., Nathues, A., Cloutis, E.A., Mann, P., Hiesinger, H., 2012. Phase reddening on near-Earth asteroids: Implications for mineralogical analysis, space weathering and taxonomic classification. Icarus 220, 36-50.

Schenk, P. et al., 2012. The geologically recent giant impact basins at Vesta's south pole. Science 336 (6082), 694-698.

Shepard, M.K., Helfenstein, P., 2007. A test of the Hapke photometric model. Theory J. Geophys. Res. 112, E03001.

Sunshine, J.M., Pieters, C.M., 1993. Estimating modal abundances from the spectra of natural and laboratory pyroxene mixtures using the modified Gaussian model. J. Geophys. Res. 98, 9075-9087.

Tholen, D.J., Hartmann, W.K., Cruikshank, D.P., 1988. 1980 PA and 1985 DO2. IAU Circ. 4655, 2.

Veeder, G.J., Hanner, M.S., Matson, D.L., Tedesco, E.F., Lebofsky, L.A., Tokunaga, A.T., 1989. Radiometry of near-Earth asteroids. Astron. J. 97, 1211-1219.

Vernazza, P. et al., 2006. Asteroid colors: A novel tool for magnetic field detection? The case of Vesta. Astron. Astrophys. 451, L43-L46.

Whiteley, R.J., 2001. A Compositional and Dynamical Survey of the Near-Earth Asteroids. PhD Thesis, University of Hawaii.

Zellner, B.H., Albrecht, R., Binzel, R.P., Gaffey, M.J., Thomas, P.C., Storrs, A.D., Wells, E.N., 1997. Hubble Space Telescope images of Asteroid 4 Vesta in 1994. Icarus $128,83-87$. 